\title{
THE PARAMETER SPACE OF CUBIC LAMINATIONS WITH A FIXED CRITICAL LEAF
}

\author{
ALEXANDER BLOKH, LEX OVERSTEEGEN, ROSS PTACEK, \\ AND VLADLEN TIMORIN
}

\begin{abstract}
Thurston parameterized quadratic invariant laminations with a non-invariant lamination, the quotient of which yields a combinatorial model for the Mandelbrot set. As a step toward generalizing this construction to cubic polynomials, we consider slices of the family of cubic invariant laminations defined by a fixed critical leaf with non-periodic endpoints. We parameterize each slice by a lamination just as in the quadratic case, relying on the techniques of smart criticality previously developed by the authors.
\end{abstract}

\section{INTRODUCTION}

One of the major goals of complex dynamics is to describe the structure of the space of complex polynomials of degree $d$ up to Moebius conjugation. In the quadratic case, this space of polynomials can be identified with the family $P_{c}(z)=z^{2}+c$ with $c \in \mathbb{C}$ parameterizing the family. Since $c$ is the critical value of $P_{c}(z)$ it shows that dynamically motivated points in the plane (i.e., the critical value of each polynomial) can be used to parameterize the space of all polynomials.

A major achievement by Thurston [Thu85] was his combinatorial interpretation of the above space. To achieve this, he introduced several important dynamical and combinatorial notions. One of them was that of a lamination. Laminations are equivalence relations on the unit circle in the plane with finite (equivalence) classes whose convex hulls in the unit disk are pairwise disjoint. A lamination is $\sigma_{2}$-invariant if its equivalence classes are preserved under $\sigma_{2}$. Using a Riemann map one can see that every quadratic polynomial with a locally connected Julia set corresponds to a unique $\sigma_{2}$-invariant lamination.

Date: January 15, 2015.

2010 Mathematics Subject Classification. Primary 37F20; Secondary 37F10, 37F50.

Key words and phrases. Complex dynamics; laminations; Mandelbrot set; Julia set.

The first author was partially supported by NSF grant DMS-1201450.

The fourth named author was partially supported by RFBR grants 13-01-12449, 13-0100969, and AG Laboratory NRU-HSE, MESRF grant ag. 11.G34.31.0023. 
Below we give a modified (but equivalent) description of Thurston's results [Thu85]. Every $\sigma_{2}$-invariant lamination $\sim$ has a unique minor set $m_{\sim}$ which is the convex hull of the image of a $\sim$-class of maximal diameter. A striking result by Thurston is that the minor sets are pairwise disjoint and form a lamination of the unit circle! The quotient space of the unit circle under this lamination then parameterizes the set of all $\sigma_{2}$-invariant laminations and serves as a model of the Mandelbrot set.

In this paper we will consider a slice of the set of all $\sigma_{3}$-laminations. Fix a chord $D=\overline{a b}$ such that $\sigma_{3}(a)=\sigma_{3}(b)$ where both $a$ and $b$ are not periodic. We consider the set $\mathrm{LAM}_{D}$ of all $\sigma_{3}$-invariant laminations $\sim$ such that $a \sim b$ except for laminations of Siegel capture type defined below. For each lamination $\sim$ in $\mathrm{LAM}_{D}$ we define its minor set $m_{\sim}$ similar to the above. Then we apply machinery from [BOPT14] to show that, similar to the case of the Mandelbrot set, the collection of all such minor sets is a lamination itself so that the quotient space of the unit circle by this lamination parameterizes $\mathrm{LAM}_{D}$.

We now describe how the paper is organized. While the precise definitions are given later, here we give heuristic versions of some of them. Also, even though all notions can be introduced for any degree $d$, our main focus here is cubic, hence some definitions are given only in the cubic case.

In Section 1 we provide the motivating background from complex dynamics, define laminations, geolaminations (which will always be denoted by $\mathcal{L}$ ), leaves, (infinite, all-critical) gaps, and related notions. In particular (cf. [BMOV13]), we define proper geolaminations as geolaminations such that no leaf can have one periodic and one non-periodic endpoint. We also review the relevant combinatorial machinery developed in [BOPT14]. Then we define less standard notions, some of which were introduced in [BOPT14]. We give an overview of these notions here. In the rest of the Introduction we will mostly consider the cubic case.

A set is a generalized (critical) quadrilateral if it is a critical leaf, an all-critical triangle (which collapses to a point), a collapsing quadrilateral (i.e., a quadrilateral which maps to a chord), or an all-critical quadrilateral (in the cubic case the last case is impossible). If $\mathcal{L}$ is cubic, a quadratically critical portrait (qc-portrait) of $\mathcal{L}$ is an ordered pair of distinct generalized quadrilaterals that are leaves or gaps of $\mathcal{L}$. In fact, cubic qc-portraits can be defined without geolaminations: an admissible (cubic) qc-portrait is an ordered pair of generalized quadrilaterals such that (1) a generalized quadrilateral with a periodic vertex either has a degenerate image or, otherwise, has a periodic edge, and (2) they and all their images intersect at most over a common edge or vertex. By a standard pullback construction an admissible cubic qc-portrait can be included in a cubic geolamination. Here we 
study only the family $\mathcal{Q C P}{ }_{3}^{n p}$ of admissible qc-portraits QCP such that the second set in QCP is a critical leaf $D$ with non-periodic endpoints.

In general, a geolamination may have no qc-portrait; it has a qc-portrait if and only if all its critical sets are collapsing quadrilaterals, critical leaves or all-critical gaps [BOPT14]. However, invariant geolaminations can be tuned to acquire a qc-portrait without compromising the dynamics. To this end, generalized quadrilaterals (which form a qc-portrait) are inserted into critical gaps of a given proper cubic geolamination $\mathcal{L}$. Using a well-known procedure, one can complete the inserted leaves and their images with their pullbacks, and in this way define a new tuned invariant geolamination. Observe that the new tuned geolamination is not necessarily proper as we may need to insert new leaves which connect periodic and non-periodic points.

Note, that here we consider proper geolaminations $\mathcal{L}$ which have a critical leaf $D$ with non-periodic endpoints. For such geolaminations there are a few possibilities concerning their critical sets (see Subsection 1.3). First, $\mathcal{L}$ can have a finite first critical set $C$. By properties of invariant geolaminations, $C$ is a gap or a leaf on which $\sigma_{3}$ acts two-to-one (unless $D$ is an edge of $C$ and so the point $\sigma_{3}(D)$ has all three of its preimages in $C$ ). Thus, if $C$ is finite, there are two cases. First, $C$ can be a $2 n+1$-gon with $D$ being one of its edges such that one can break down all its edges into pairs of "sibling edges", i.e. leaves with the same image except for $D$ (one can say that the "sibling edge" of $D$ is the vertex of $C$ not belonging to $D$ and with the same image as $D$ ). Second, $C$ can be a $2 m$-gon such that $D$ is not an edge of $C$ in which case $\left.\sigma_{3}\right|_{C}$ is two-to-one.

Also, $C$ could be an infinite gap. Then there are two cases. First, $C$ may be a periodic Fatou gap of some period $k$ and degree 2 (observe that in this case $D$ may well be an edge of $C$ ). Second, there may exist a periodic Siegel gap $U$ with $D$ being one of its edges and an infinite gap $V$ such that $\sigma_{3} \mid \operatorname{Bd}(V)$ is two-to-one and $V$ eventually maps to $U$. We call such (geo)laminations Siegel (geo)laminations of capture type.

Now, let $\mathbb{L} \mathbb{P}_{3}^{n p}$ be the family of all cubic proper geolaminations which have a critical leaf with non-periodic endpoints except for Siegel geolaminations of capture type. If $\mathcal{L} \in \mathbb{L} \mathbb{P}_{3}^{n p}$ has a critical leaf $D$ with non-periodic endpoints, then a qc-portrait $\mathrm{QCP}=(Q, D)$ is called privileged for $\mathcal{L}$ if and only if $Q \subset C$ where $C \neq D$ is a critical set of $\mathcal{L}$ and either $C$ is finite, or $C$ is a periodic Fatou gap of degree two and period $k$ and $Q$ is a collapsing quadrilateral which is the convex hull of a (possibly degenerate) edge $\ell$ of $C$ of period $k$ and another edge $\hat{\ell}$ of $C$ such that $\sigma_{3}(\ell)=\sigma_{3}(\hat{\ell})$.

In Section 2 we state our main results. We show that for each $\mathcal{L} \in \mathbb{L} \mathbb{P}_{3}^{n p}$ there are only finitely many privileged qc-portraits and for every admissible qc-portrait $\mathrm{QCP} \in \mathcal{Q C} \mathcal{P}_{3}^{n p}$, there exists $\mathcal{L}_{\mathrm{QCP}} \in \mathbb{L P}_{3}^{n p}$ such that $\mathrm{QCP}$ is 
privileged for $\mathcal{L}_{\mathrm{QCP}}$ and the lamination induced by $\mathcal{L}_{\mathrm{QCP}}$ is unique. For a critical leaf $D$ with non-periodic endpoints, $\mathcal{S}_{D}$ denotes the collection of all admissible qc-portraits $(Q, D)$ of $\mathcal{Q C} \mathcal{P}_{3}^{n p}$ with $D$ as the second element. To each such qc-portrait $(Q, D)$ we associate the set (a chord or a point called minor) $\sigma_{3}(Q) \subset \overline{\mathbb{D}}$. For each such chord we identify its endpoints, extend this identification by transitivity and define the corresponding equivalence relation $\sim_{D}$ on $\mathbb{S}$. Our main result is that $\sim_{D}$ is itself a lamination whose quotient is a parameterization of $\mathrm{LAM}_{D}$.

Section 3 describes how we will tag $\sigma_{3}$-invariant geolaminations with privileged qc-portraits and shows that the tagging generalizes Thurston's tagging of $\sigma_{2}$-invariant geolaminations by minors. We show that any qcportrait which contains a critical leaf without periodic endpoints is privileged for some geolamination. This implies that when we fix a critical leaf with non-periodic endpoints we obtain a closed set of qc-portraits.

In Section 4 we study possible intersections of minors of our qc-portraits. Finally, in Section 5 we construct the parameter lamination $\sim_{D}$.

\section{PRELIMINARIES}

We assume basic knowledge of complex dynamics and use standard notation. Let $P: \mathbb{C} \rightarrow \mathbb{C}$ be a polynomial of degree $d \geq 2, A_{\infty}$ be the basin of attraction of infinity, and $J_{P}=\operatorname{Bd}\left(A_{\infty}\right)$ be the Julia set of $P$. When $J_{P}$ is connected, $A_{\infty}$ is simply connected and conformally isomorphic to $\mathbb{C} \backslash \mathbb{D}$ by a unique isomorphism $\phi: \mathbb{C} \backslash \overline{\mathbb{D}} \rightarrow A_{\infty}$ asymptotic to the identity at $\infty$. By a theorem of Böttcher (see, e.g., [Mil00, Theorem 9.1]), $\phi$ conjugates $\left.P\right|_{A_{\infty}}$ with $\left.z^{d}\right|_{\mathbb{C} \backslash \overline{\mathbb{D}}}$. If $J_{P}$ is locally connected, then $\phi$ extends continuously to a semiconjugacy $\bar{\phi}$ between $\sigma_{d}=\left.z \mapsto z^{d}\right|_{\mathbb{S}}$ and $\left.P\right|_{J_{P}}$ :

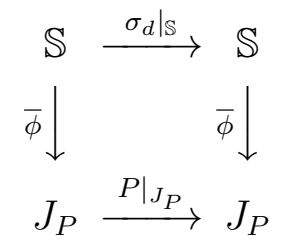

The lamination generated by $P$ is the equivalence relation $\sim_{P}$ on $\mathbb{S}$ whose classes are $\bar{\phi}$-fibers, i.e. point-preimages under $\bar{\phi}$. We call $\widehat{J}_{P}=\mathbb{S} / \sim_{P}$ a topological Julia set and $\widehat{P}$, the corresponding map induced on $\widehat{J}_{P}$ by $\sigma_{d}$, a topological polynomial. Evidently $\left.P\right|_{J_{P}}$ and $\left.\widehat{P}\right|_{\widehat{J_{P}}}$ are conjugate. The collection $\mathcal{L}_{P}$ of chords of $\overline{\mathbb{D}}$ which are edges of convex hulls of $\sim_{P}$ classes is called the geolamination generated by $P$. 
1.1. Laminations, Geolaminations, and their Properties. Laminations and geolaminations can be defined independently of polynomials. This approach is due to Thurston [Thu85] who constructed a combinatorial model of the Mandelbrot set by parameterizing quadratic geolaminations. We define laminations to be equivalence relations without underlying dynamics.

Definition 1.1 (Laminations). An equivalence relation $\sim$ on the unit circle $\mathbb{S}$ is called a lamination if either $\mathbb{S}$ is one $\sim$-class (such laminations are called degenerate), or the following holds:

(E1) the graph of $\sim$ is a closed subset of $\mathbb{S} \times \mathbb{S}$;

(E2) the convex hulls of distinct equivalence classes are disjoint;

(E3) each equivalence class of $\sim$ is finite.

These properties are satisfied by laminations generated by polynomials. Property (E1) is necessary for $\mathbb{S} / \sim$ to be a metric space, (E2) follows from the semiconjugacy $\bar{\phi}$ (a proof can be found in [Sch09, Proposition II.3.3]), and (E3) follows from [Mil00, Lemma 18.12] for (pre)periodic points and from Kiwi ([Kiw02] $)$ for points with infinite orbit. Definition 1.2 enforces dynamics on laminations consistent with those generated by polynomials.

Definition 1.2 (Laminations and dynamics). An equivalence relation $\sim$ is called $\left(\sigma_{d^{-}}\right)$invariant if:

(D1) $\sim$ is forward invariant: for a $\sim$-class $\mathfrak{g}$, the set $\sigma_{d}(\mathfrak{g})$ is a $\sim$-class;

(D2) for any $\sim$-class $\mathfrak{g}$, the map $\sigma_{d}: \mathfrak{g} \rightarrow \sigma_{d}(\mathfrak{g})$ extends to $\mathbb{S}$ as an orientation preserving covering map such that $\mathfrak{g}$ is the full preimage of $\sigma_{d}(\mathfrak{g})$ under this covering map.

There is a useful geometric object associated with each lamination.

Definition 1.3. For a lamination $\sim$, take convex hulls of all its classes and consider the family of chords which are their edges (together with all single points of the unit circle). This family of (possibly degenerate) chords is called a geolamination generated by $\sim$ and is denoted by $\mathcal{L}_{\sim}$.

Definition 1.3 makes sense for non-invariant and invariant laminations; for invariant laminations we use notation described in Definition 1.4 .

Definition 1.4. The family of all invariant geolaminations generated by laminations is denoted by $\mathbb{L} \mathcal{Q}$; if we consider only $\sigma_{d}$-invariant laminations, the corresponding family of geolaminations is denoted by $\mathbb{L} \mathcal{Q}_{d}$. Geolaminations from $\mathbb{L} \mathcal{Q}\left(\mathbb{L} \mathcal{Q}_{d}\right)$ are called $\mathbb{L} \mathcal{Q}$-geolaminations ( $\mathbb{L} \mathcal{Q}_{d^{-}}$geolaminations).

Similar notions were defined by Thurston in [Thu85] with no equivalence relations involved. For a collection $\mathcal{R}$ of chords of $\mathbb{D}$ set $\mathcal{R}^{+}=\bigcup \mathcal{R}$. 
Definition 1.5 (Geolaminations). Two chords of $\overline{\mathbb{D}}$ are called unlinked if they are disjoint in $\mathbb{D}$. A geolamination is a collection $\mathcal{L}$ of (perhaps degenerate) pairwise unlinked chords of $\overline{\mathbb{D}}$ called leaves such that $\mathcal{L}^{+}$is closed, and all points of $\mathbb{S}$ are elements of $\mathcal{L}$. A gap of $\mathcal{L}$ is the closure of a component of $\mathbb{D} \backslash \mathcal{L}^{+}$. If $\mathcal{L}=\mathcal{L}_{\sim}$ is a geolamination generated by a lamination $\sim$, gaps of $\mathcal{L}_{\sim}$ will also be called gaps of $\sim$. A gap $G$ is finite (infinite) depending on whether $G \cap \mathbb{S}$ is finite (infinite). Gaps $G$ such that $G \cap \mathbb{S}$ is uncountable, are called Fatou gaps. For Fatou gaps $G$ define the monotone map $\psi_{G}: \operatorname{Bd}(G) \rightarrow \mathbb{S}$ which collapses all edges of $G$ to points.

If $x, y \in \mathbb{S}$ are the endpoints of a chord $\ell$, set $\ell=\overline{x y}$. Given a closed set $A \subset \mathbb{S}$, let $\sigma_{d}(\mathrm{CH}(A))=\mathrm{CH}\left(\sigma_{d}(A)\right)$ (in particular, $\sigma_{d}(\overline{x y})=\overline{\sigma_{d}(x) \sigma_{d}(y)}$ ). Any non-degenerate chord $\ell$ such that $\sigma_{d}(\ell)$ is a point is called critical. Given a geolamination $\mathcal{L}$, we linearly extend $\sigma_{d}$ over leaves of $\mathcal{L}$; clearly, this extension is continuous and well-defined. With this extension, we can define $\sigma_{d}$-invariant geolaminations. Here we rely on [BMOV13] where the definition is a bit different from Thurston's [Thu85].

Definition 1.6 (Invariant sibling geolaminations [ $[\mathrm{BMOV13}]$ ). A geolamination $\mathcal{L}$ is $\left(\sigma_{d^{-}}\right)$invariant (sibling) geolamination provided:

(1) for each $\ell \in \mathcal{L}$, we have $\sigma_{d}(\ell) \in \mathcal{L}$,

(2) for each $\ell \in \mathcal{L}$ there exists $\ell^{*} \in \mathcal{L}$ so that $\sigma_{d}\left(\ell^{*}\right)=\ell$.

(3) for each $\ell \in \mathcal{L}$ such that $\sigma_{d}(\ell)$ is non-degenerate, there exist $d$ pairwise disjoint leaves $\ell_{1}, \ldots, \ell_{d}$ in $\mathcal{L}$ so that $\ell=\ell_{1}$ and $\sigma_{d}\left(\ell_{i}\right)=$ $\sigma_{d}(\ell)$ for all $i=1, \ldots, d$.

If a geolamination satisfies only condition 1 , we call it a forward $\left(\sigma_{d^{-}}\right.$ )invariant sibling geolamination.

Any leaf $\ell^{*}$ with $\sigma_{d}\left(\ell^{*}\right)=\ell$ is called a pullback of $\ell$. Also, two leaves with the same $\sigma_{d}$-image are called siblings, and the leaves $\ell_{1}=\ell, \ldots, \ell_{d}$ in (3) are said to form a disjoint sibling collection of $\ell$ [BMOV13]. The space of all $\sigma_{d}$-invariant sibling geolaminations is denoted $\mathbb{L}_{d}$.

Thurston defines $\sigma_{d}$-invariant geolaminations differently. He requires that geolaminations satisfy (1) above, be such that each leaf $\ell$ has $d$ disjoint pullbacks, and be gap invariant.

Definition 1.7 (Gap invariance [Thu85]). Suppose that for any gap $G$ of a geolamination $\mathcal{L}$ the set $H=\sigma_{d}(G)$ is either a point of $\mathbb{S}$, a leaf of $\mathcal{L}$, or a gap of $\mathcal{L}$. Moreover, if $H$ is a gap then $\sigma_{d}: \operatorname{Bd}(G) \rightarrow \operatorname{Bd}(H)$ is the positively oriented composition of a monotone map and a covering map. Then $\mathcal{L}$ is said to be gap invariant.

Let us now define the degree of $\sigma_{d}$ on a gap or leaf of $\mathcal{L}$. 
Definition 1.8 (Degree of $\sigma_{d}$ on a gap or leaf). Let $G$ be a gap or leaf of a gap invariant geolamination and let $H=\sigma_{d}(G)$. Then the degree of $\left.\sigma_{d}\right|_{G}$ is either the cardinality of $G \cap \mathbb{S}$ (if $H$ is a point), or, otherwise, the number of components of the set $\sigma_{d}^{-1}(x) \cap \operatorname{Bd}(G)$ where $x$ is any point of $\operatorname{Bd}(H)$.

It is easy to see that the degree of $\left.\sigma_{d}\right|_{G}$ does not depend on the choice of the point $x \in \operatorname{Bd}(H)$. In case when $\sigma_{d}(G)$ is a gap, the degree of $\left.\sigma_{d}\right|_{G}$ can be defined as the degree of a covering map involved in the representation of $\left.\sigma_{d}\right|_{\operatorname{Bd}(G)}$ as the composition of a monotone map and a covering map.

Definition 1.9. A gap $G$ all of whose edges are critical is said to be allcritical. A gap $G$ is called critical if the degree of $\left.\sigma_{d}\right|_{G}$ is greater than 1 .

Definition 1.6 conveniently deals only with leaves. This leads to useful results on properties of $\sigma_{d}$-invariant sibling geolaminations [BMOV13]. We will agree to endow any family of compact subsets of $\overline{\mathbb{D}}$ with the Hausdorff metric and the induced topology. In particular, we define a natural topology on $\mathbb{L}_{d}$ induced by the Hausdorff metric on subcontinua $\mathcal{L}^{+}$of $\overline{\mathbb{D}}$ where $\mathcal{L} \in$ $\mathbb{L}_{d}$ (from now on talking about $\mathbb{L}_{d}$ we mean the just defined topological space rather than a family of geolaminations).

Theorem 1.10 ([BMOV13] $)$. The following results hold:

(1) $\mathbb{L} \mathcal{Q}_{d} \subset \mathbb{L}_{d}$ (i.e., geolaminations generated by $\sigma_{d}$-invariant laminations are $\sigma_{d}$-invariant sibling geolaminations);

(2) $\mathbb{L}_{d}$ is compact (thus, $\mathbb{L}_{d}$ contains all limits of geolaminations from $\left.\mathbb{L} \mathcal{Q}_{d}\right)$;

(3) $\sigma_{d}$-invariant sibling geolaminations are $\sigma_{d}$-invariant in the sense of Thurston.

We will also need a few technical results of [BMOV13]. Denote by $<$ the positive (circular) order on $\mathbb{S}$. That is, given points $x, y, z \in \mathbb{S}, x<y<z$ if the counterclockwise arc of $\mathbb{S}$ from $x$ to $y$ contains $y$ in its interior. A consequence of gap invariance is that if an arc in the boundary of a gap of a $\sigma_{d}$-invariant geolamination is mapped injectively by $\sigma_{d}$, then $\sigma_{d}$ preserves the (circular) order of points of that arc. In [BMOV13] this property was explored for leaves of $\sigma_{d}$-invariant sibling geolaminations emanating from the same point of $\mathbb{S}$ which may or may not be edges of a common gap.

Lemma 1.11 ([BMOV13], Lemma 3.7). Let $\mathcal{L}$ be a $\sigma_{d}$-invariant sibling geolamination and $T \subset \mathcal{L}^{+}$be an arc consisting of two leaves with a common endpoint or a triod consisting of three leaves with a common endpoint. Suppose that $Y \subset \mathcal{L}^{+}$is an arc (triod) such that $\sigma_{d}(Y)=T$ and $\left.\sigma_{d}\right|_{Y}$ is one-to-one. Then $\left.\sigma_{d}\right|_{Y \cap \mathbb{S}}$ preserves circular order.

Corollary 1.12 easily follows from Lemma 1.11 . 
Corollary 1.12. Let $\mathcal{L}$ be a $\sigma_{d}$-invariant sibling geolamination. Let $x<$ $y<z<x$ be the endpoints of two non-critical leaves $\overline{x y}$ and $\overline{y z}$ of $\mathcal{L}$ such that $\sigma_{d}(x)=\sigma_{d}(z)$. If now $z<t<x$ and $\overline{y t}$ is a leaf of $\mathcal{L}$ then either $\sigma_{d}(t)=\sigma_{d}(y)$ or $\sigma_{d}(t)=\sigma_{d}(x)$; in particular, there are at most $2 d-3$ such leaves $\overline{y t}$.

Proof. Suppose otherwise. Then if we apply Lemma 1.11 to $\overline{x y} \cup \overline{y t}$ we see that $\sigma_{d}(x)<\sigma_{d}(y)<\sigma_{d}(t)<\sigma_{d}(x)$. On the other hand, if we apply Lemma 1.11 to $\overline{y z} \cup \overline{y t}$ we see that $\sigma_{d}(y)<\sigma_{d}(z)<\sigma_{d}(t)<\sigma_{d}(y)$. Since $\sigma_{d}(x)=\sigma_{d}(z)$, this is a contradiction.

Corollary 1.13 follows from Corollary 1.12 . By definition, if a noncritical leaf $\ell$ of a $\sigma_{d}$-invariant sibling geolamination has overall $d-1$ other leaves of $\mathcal{L}$ which map to $\sigma_{d}(\ell)$, then all these $d$ leaves are pairwise disjoint and form a unique disjoint sibling collection of leaves with image $\sigma_{d}(\ell)$.

Corollary 1.13. There are finitely many leaves $\ell$ such that more than $d$ leaves have the image $\sigma_{d}(\ell)$.

Proof. We may assume that $\ell$ is non-critical. If $\ell$ has more than $d-1$ other leaves with the image $\sigma_{d}(\ell)$ then at least two of them will share an endpoint (say, $y$ ) with the two other endpoints (say, $x$ and $z$ ) mapped to the same point. Set $\mathrm{CH}(x, y, z)=T_{\ell}$. Call $\overline{x z}$ the base of $T_{\ell}$ and call $y$ the top vertex of $T_{\ell}$. It is easy to see that there are at most finitely many (in fact, no more than $2 d-3$ ) triangles whose bases are pairwise unlinked; let $T^{1}, \ldots, T^{k}$ be a maximal collection of such triangles with pairwise unlinked bases. Any remaining triangle $T_{\ell}$ is such that its base crosses the base of some triangle $T^{j}, 1 \leq j \leq k$. Since sides of all these triangles which are not their bases are unlinked leaves, then $T_{\ell}$ and $T^{j}$ must share a vertex. Clearly, the number of vertices of triangles $T^{1}, \ldots, T^{k}$ is finite. By Corollary 1.12 , each of them can be the top vertex of finitely many triangles $T_{\ell}$. Hence the overall number of such triangles is finite as desired.

This helps in studying disjoint sibling collections.

Lemma 1.14. If $\ell$ is a non-critical non-isolated leaf, then there exists a disjoint sibling collection which contains $\ell$ and consists of non-isolated leaves. I.e., if $\hat{\ell}$ is a non-degenerate non-isolated from one (two) sides leaf then there is a disjoint sibling collection of leaves mapped to $\hat{\ell}$ such that leaves in this collection are non-isolated from the appropriate side( $s)$.

Proof. Let $\ell_{i}^{1} \rightarrow \ell^{1}=\ell$ be a sequence of leaves which converge to $\ell$ from one side. By Corollary 1.13 we may assume that for each $i$ the leaf $\ell_{i}$ is a member of a disjoint sibling collection $L_{i}=\left\{\ell_{i}^{1}, \ell_{i}^{2}, \ldots, \ell_{i}^{d}\right\}$ which includes all leaves mapped to $\sigma_{d}\left(\ell_{i}^{1}\right)$. By compactness we may assume that leaves 
in these collections are ordered so that for each $1 \leq j \leq d, \lim _{i} \ell_{i}^{j}=\ell^{j}$. Clearly, $\sigma_{d}\left(\ell^{j}\right)=\sigma_{d}(\ell)$ and all leaves $\ell^{j}$ are non-isolated.

To show that all these leaves are pairwise disjoint, observe that since the leaves $\ell_{i}^{1}$ approach $\ell^{1}$ from one side, then for any $j$, the leaves $\ell_{i}^{j}$ approach $\ell^{j}$ from one side. Thus, the leaves $\sigma_{d}\left(\ell_{i}^{j}\right)$ approach $\sigma_{d}\left(\ell^{j}\right)$ from one side too. If now, say, $\ell^{2}=\ell^{3}$ then it would follow that leaves $\ell_{i}^{2}, \ell_{i}^{3}$ are disjoint, close to each other, and have the same image, which is impossible. Suppose that $\ell^{2} \neq \ell^{3}$ come out of the same point but are otherwise disjoint. Then the leaves $\ell^{2}$ and $\ell^{3}$ form a wedge, and the leaves $\ell_{i}^{2}, \ell_{i}^{3}$ are located outside the wedge close to the appropriate sides of the wedge (recall that all these leaves come from the same geolamination and are therefore pairwise unlinked). Then their images approach $\sigma_{d}\left(\ell^{1}\right)$ from opposite sides, a contradiction.

Lemma 1.14 allows us to apply a certain "cleaning" procedure to construct other $\sigma_{d}$-invariant sibling geolaminations out of a given geolamination.

Theorem 1.15. Let $\mathcal{L}$ be a $\sigma_{d}$-invariant sibling geolamination and $\mathcal{A}$ be a family of grand orbits of its leaves. Construct a collection of leaves $\mathcal{L}^{\prime}$ by removing from $\mathcal{L}$ all isolated leaves belonging to $\mathcal{A}$. Then $\mathcal{L}^{\prime}$ is a $\sigma_{d^{-}}$ invariant sibling geolamination.

Proof. Since non-isolated leaves map to non-isolated leaves then $\mathcal{L}^{\prime}$ is forward invariant. Let $\ell^{\prime} \in \mathcal{L}^{\prime}$ be non-degenerate. Then either $\ell^{\prime} \notin \mathcal{A}$, or $\ell^{\prime} \in \mathcal{A}$ is non-isolated. In the former case by definition there is a leaf $\ell^{\prime \prime} \in \mathcal{L}$ such that $\sigma_{d}\left(\ell^{\prime \prime}\right)=\ell^{\prime}$, and since $\ell^{\prime} \notin \mathcal{A}$ it follows that $\ell^{\prime \prime} \notin \mathcal{A}$ and hence $\ell^{\prime \prime} \in \mathcal{L}^{\prime}$. In the latter case the claim follows from Lemma 1.14 . Thus, any non-degenerate leaf from $\mathcal{L}^{\prime}$ has a preimage in $\mathcal{L}^{\prime}$. Finally let $\ell^{\prime} \in \mathcal{L}^{\prime}$ be non-critical. Then again either $\ell^{\prime} \notin \mathcal{A}$, or $\ell^{\prime} \in \mathcal{A}$ is non-isolated. In the former case the claim is obvious as all disjoint sibling collections of $\ell^{\prime}$ are disjoint from $\mathcal{A}$. In the latter case the claim follows from Lemma 1.14

Arguments, similar to those in the proof of Corollary 1.12 and based upon Lemma 1.11, prove Corollary 1.16, we leave the proof to the reader.

Corollary 1.16. If $\mathcal{L}$ is a $\sigma_{d}$-invariant sibling geolamination and $\overline{a b}$ is a leaf of $\mathcal{L}$ with periodic endpoints then a and b are periodic of the same period.

The paper [BMOV13] also explored how disjoint sibling collections must be located in $\overline{\mathbb{D}}$. To state the corresponding lemma we need a notational agreement. If $X$ is a collection of pullbacks of a leaf $\overline{a b}$, we denote the endpoints of chords of $X$ by the same letters as for the endpoints of their images but with a hat and distinct subscripts, and call them correspondingly ( $a$-points, $b$-points etc). Lemma 1.17 is typically used in the situation of Figure 1, when no leaves cross the critical chord joining two sibling leaves. 
We say that two distinct chords cross each other, or are linked, if they intersect inside the open unit disk $\mathbb{D}$.

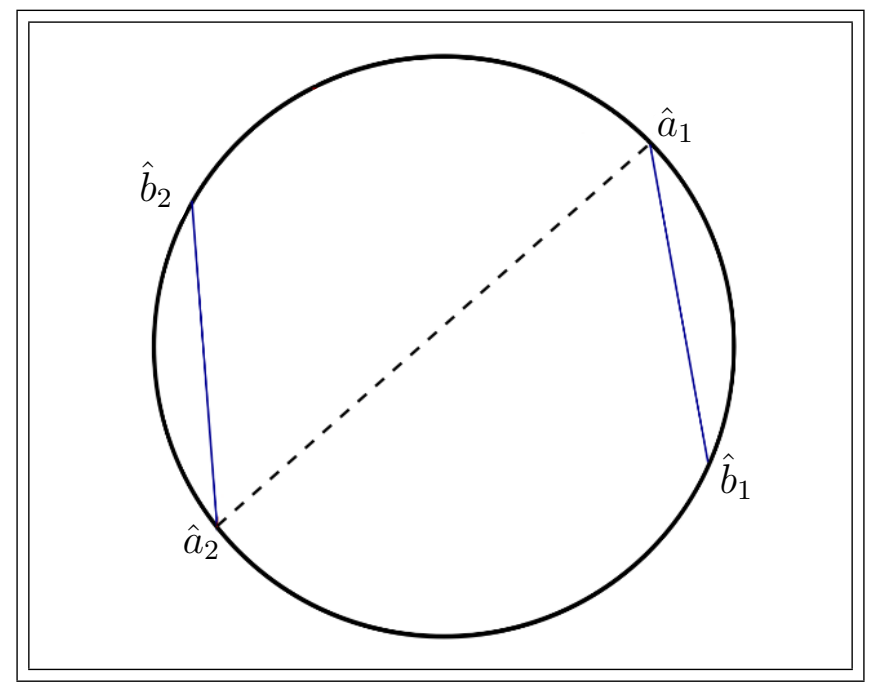

FIGURE 1. Siblings and critical leaves.

Siblings must be on opposite sides of the chord $\overline{\hat{a}_{1} \hat{a}_{2}}$ which is not crossed by leaves of the disjoint sibling collection.

Lemma 1.17 ([BMOV13], Lemma 3.8). Let $X$ be a disjoint sibling collection of leaves mapped to a leaf $\overline{a b}$ and $\overline{\hat{a}_{1} \hat{b}_{1}}, \overline{\hat{a}_{2} \hat{b}_{2}}$ be two leaves from $X$. Then the number of leaves from $X$ crossing the chord $\overline{\hat{a}}_{1} \hat{a}_{2}$ inside $\mathbb{D}$ is even if and only if either $\hat{a}_{1}<\hat{b}_{1}<\hat{a}_{2}<\hat{b}_{2}$ or $\hat{a}_{1}<\hat{b}_{2}<\hat{a}_{2}<\hat{b}_{1}$. In particular, if there exists a concatenation $Q$ of chords connecting $\hat{a}_{1}$ and $\hat{a}_{2}$, disjoint with leaves of $X$ except the points $\hat{a}_{1}, \hat{a}_{2}$, then either $\hat{a}_{1}<\hat{b}_{1}<\hat{a}_{2}<\hat{b}_{2}$ or $\hat{a}_{1}<\hat{b}_{2}<\hat{a}_{2}<\hat{b}_{1}$.

We will also need the following easy corollary of Lemma 1.17 .

Corollary 1.18. Let $X$ be a disjoint sibling collection of leaves mapped to a leaf $\overline{a b}$ and $\overline{\hat{a}_{1} \hat{b}_{1}}, \overline{\hat{a}_{2} \hat{b}_{2}}$ be two leaves from $X$. Suppose that there exists a concatenation $Q$ of pairwise non-crossing critical chords connecting $\hat{a}_{1}$ and $\hat{a}_{2}$, with endpoints separated by $\overline{\hat{a}_{1} \hat{a}_{2}}$ from $\hat{b}_{1}$ inside $\mathbb{D}$, and disjoint from the leaves of $X$ except for their endpoints. Then either $\hat{a}_{1}<\hat{b}_{1}<\hat{a}_{2}<\hat{b}_{2}$ or $\hat{a}_{1}<\hat{b}_{2}<\hat{a}_{2}<\hat{b}_{1}$.

Proof. Without loss of generality, assume that $\hat{a}_{1}<\hat{a}_{2}<\hat{b}_{1}$. By the assumptions of the lemma there is a chain $Y$ of pairwise non-crossing critical chords $\overline{\hat{a}_{1} x_{1}}, \overline{x_{1} x_{2}}, \ldots, \overline{x_{n} \hat{a}_{2}}$ with $\hat{a}_{1}<x_{1}<x_{2}<\cdots<x_{n}<\hat{a}_{2}$ each of which does not cross leaves from $X$. By definition, $X$ must contain $n$ 
disjoint leaves with endpoints $x_{1}, \ldots, x_{n}$ respectively. Moreover, applying Lemma 1.17 step by step and using the fact that chords in $Y$ do not cross chords of $X$ we see that $X$ contains leaves $\overline{y_{1} x_{1}}, \ldots, \overline{y_{n} x_{n}}$ such that

$$
\hat{a}_{1}<y_{1}<x_{1}<y_{2}<x_{2}<\cdots<y_{n}<x_{n}<\hat{b}_{2}<\hat{a}_{2}
$$

which implies that $\hat{a}_{1}<\hat{b}_{2}<\hat{a}_{2}<\hat{b}_{1}$ as desired.

One benefit of working with geolaminations is the ability to construct $\sigma_{d}$-invariant geolaminations from a small generating set. This is due to Thurston [Thu85] for his definition of $\sigma_{d}$-invariance, but is easily modified for $\sigma_{d}$-invariant sibling geolaminations. The geolamination of Theorem 1.19 is constructed by iteratively adding preimages of leaves of $\widehat{\mathcal{L}}$ and taking the closure after countably many steps.

Theorem 1.19 (cf [Thu85]). Let $\widehat{\mathcal{L}}$ be a forward $\sigma_{d}$-invariant sibling geolamination. Then there exists a $\sigma_{d}$-invariant geolamination $\mathcal{L} \supset \widehat{\mathcal{L}}$ for which grand orbits of leaves of $\widehat{\mathcal{L}}$ are dense.

In what follows we call $\sigma_{2}$-invariant ( $\sigma_{3}$-invariant) sibling geolaminations respectively quadratic and cubic (sibling) geolaminations.

1.2. Accordions and Linked Geolaminations. By [Thu85], a quadratic geolamination $\mathcal{L}$ has one or two longest leaves called majors of $\mathcal{L}$. If the major is unique then it must be a diameter, otherwise the two majors are disjoint. In any case, the majors are denoted $M_{\mathcal{L}}$ and $M_{\mathcal{L}}^{\prime}$. The minor $m_{\mathcal{L}}$ of $\mathcal{L}$ is defined as $m_{\mathcal{L}}=\sigma_{2}\left(M_{\mathcal{L}}\right)=\sigma_{2}\left(M_{\mathcal{L}}^{\prime}\right)$. Thurston proves that no two minors intersect in $\mathbb{D}$ and the collection QML of all quadratic minors is a geolamination itself called the Quadratic Minor Lamination.

In [BOPT14], we partially generalize this result to cubic geolaminations. Using minors alone for such geolaminations is impossible. While in the quadratic case $m_{\mathcal{L}}$ is uniquely pulled back under $\sigma_{2}$ to a pair of majors, there are multiple ways to pull leaves back under $\sigma_{3}$ which result in very different geolaminations. To avoid this ambiguity, we associate cubic geolaminations with ordered pairs of generalized critical quadrilaterals; such pairs are called qc-portraits. In the case of $\sigma_{2}$, this is equivalent to associating to a quadratic geolamination $\mathcal{L}$ the set $\mathrm{CH}\left(\sigma_{2}^{-1}\left(m_{\mathcal{L}}\right)\right)$.

Definition 1.20 (Generalized critical quadrilaterals). A generalized quadrilateral $Q$ is the circularly ordered 4-tuple $\left[a_{0}, a_{1}, a_{2}, a_{3}\right]$ of marked points $a_{0} \leq a_{1} \leq a_{2} \leq a_{3} \leq a_{0}$ in $\mathbb{S}$. Such $Q$ is said to be $\left(\sigma_{d^{-}}\right)$critical if $\overline{a_{0} a_{2}}$ and $\overline{a_{1} a_{3}}$ are non-degenerate $\sigma_{d}$-critical chords (called spikes). Generalized $\sigma_{d}$-critical quadrilaterals $\left[a_{0}, a_{1}, a_{2}, a_{3}\right],\left[a_{1}, a_{2}, a_{3}, a_{0}\right]$, and the other circular permutations of the vertices, are viewed as equal. The chords $\overline{a_{0} a_{1}}, \overline{a_{1} a_{2}}, \overline{a_{2} a_{3}}, \overline{a_{3} a_{0}}$ are called edges of $Q$. 
Note that a generalized critical quadrilateral $Q$ is either a critical leaf, or an all-critical triangle, or an all-critical quadrilateral(impossible if the degree is three), or a quadrilateral which collapses to a leaf (a collapsing quadrilateral). Although the definition of a qc-portrait is suitable for $\sigma_{d^{-}}$ invariant sibling geolaminations, we simplify it here for $\sigma_{3}$. When saying that a geolamination $\mathcal{L}$ has (or is with) a generalized critical quadrilateral $Q$ we mean that $Q$ is a leaf or a gap of $\mathcal{L}$.

Definition 1.21 (Quadratic criticality). Let $(\mathcal{L}, \mathrm{QCP})$ be a cubic geolamination with an ordered pair QCP of distinct generalized critical quadrilaterals which do not intersect in $\mathbb{D}$ other than over a common edge/vertex. Then QCP is called a quadratically critical portrait (qc-portrait) for $\mathcal{L}$ while the pair $(\mathcal{L}, \mathrm{QCP})$ is called a geolamination with qc-portrait.

Note that we do not require that the two generalized critical quadrilaterals be disjoint. In fact, one may even be a subset of the other. However we do require that they be distinct. Thus, a critical leaf repeated twice is not a qc-portrait.

In the quadratic case the notion of a qc-portrait reduces to that of a critical leaf or collapsing quadrilateral(recall, that a collapsing quadrilateral is a critical quadrilateral whose image is a non-degenerate chord). In this case the image of a collapsing quadrilateral or a critical leaf of a quadratic geolamination $\mathcal{L}$ is the minor $m_{\mathcal{L}}$ of $\mathcal{L}$.

Now we discuss strong linkage between generalized critical quadrilaterals and then linkage between cubic geolaminations with qc-portraits. For a generalized quadrilateral $Q$, call a component of $\mathbb{S} \backslash Q$ a hole of $Q$.

Definition 1.22 (Strongly linked quadrilaterals). Let $A$ and $B$ be generalized quadrilaterals. Say that $A$ and $B$ are strongly linked if the vertices of $A$ and $B$ can be numbered so that

$$
a_{0} \leq b_{0} \leq a_{1} \leq b_{1} \leq a_{2} \leq b_{2} \leq a_{3} \leq b_{3} \leq a_{0}
$$

where $a_{i}, 0 \leq i \leq 3$, are vertices of $A$ and $b_{i}, 0 \leq i \leq 3$ are vertices of $B$. Note that the vertices of the two quadrilaterals here need not strictly alternate on the circle.

Definition 1.23 is a cubic version of the corresponding one from [BOPT14].

Definition 1.23 (Linked qc-portraits). Let qc-portraits $\mathrm{QCP}_{1}=\left(C_{1}^{1}, C_{1}^{2}\right)$ and $\mathrm{QCP}_{2}=\left(C_{2}^{1}, C_{2}^{2}\right)$ be such that for every $1 \leq i \leq 2$ the sets $C_{1}^{i}$ and $C_{2}^{i}$ are either strongly linked generalized critical quadrilaterals or share a spike. Then the qc-portraits are said to be linked while the critical sets $C_{1}^{i}$ and $C_{2}^{i}, i=1,2$ are called associated. If $\left(\mathcal{L}_{1}, \mathrm{QCP}_{1}\right)$ and $\left(\mathcal{L}_{2}, \mathrm{QCP}_{2}\right)$ are geolaminations with qc-portraits then they are called linked if either $\mathrm{QCP}_{1}$ and $\mathrm{QCP}_{2}$ are linked, or $\mathcal{L}_{1}$ and $\mathcal{L}_{2}$ share an all-critical triangle. 
Observe that in [BOPT14] geolaminations $\mathcal{L}_{1}, \mathcal{L}_{2}$ from Definition 1.23, for which either both pairs of associated generalized critical quadrilaterals in qc-portraits share a spike or $\mathcal{L}_{1}, \mathcal{L}_{2}$ themselves share an all-critical triangle, are said to be essentially equal rather than linked. However for our purposes this fine distinction does not matter much, thus in this paper we use a little less precise definition above.

Thurston's result that no quadratic geolaminations have linked minors is equivalent to the statement that there are no quadratic geolaminations with linked qc-portraits. Also, in [BOPT14] the notion of linked geolaminations was introduced and various results were obtained in the degree $d$ case; the results stated in the rest of this subsection are restatements of the results of [BOPT14] for the cubic case. The main tool used in [BOPT14] to study linked $\sigma_{d}$-invariant sibling geolaminations with qc-portraits is an accordion designed to track linked leaves from linked geolaminations.

Definition 1.24. Let $\ell_{1}, \ell_{2}$ be leaves of $\sigma_{d}$-invariant sibling geolaminations $\mathcal{L}_{1}, \mathcal{L}_{2}$. Denote by $A_{\ell_{2}}\left(\ell_{1}\right)$ the collection of leaves from the forward orbit of $\ell_{2}$ linked with $\ell_{1}$ together with $\ell_{1}$. We call $A_{\ell_{2}}\left(\ell_{1}\right)$ the accordion of $\ell_{1}$ with respect to $\ell_{2}$. Abusing notation, we often denote $A_{\ell_{2}}^{+}\left(\ell_{1}\right)$ by $A_{\ell_{2}}\left(\ell_{1}\right)$.

An important property of accordions is introduced in Definition 1.25 .

Definition 1.25. Say that $\ell_{1}$ has order preserving accordions with respect to $\ell_{2}$ if $A_{\ell_{2}}\left(\ell_{1}\right) \neq\left\{\ell_{1}\right\}$ and for all $k \geq 0$, the restriction of $\sigma_{d}$ to $A_{\ell_{2}}\left(\sigma_{d}^{k}\left(\ell_{1}\right)\right) \cap \mathbb{S}$ is order preserving. If $\ell_{1}$ has order preserving accordions with respect to $\ell_{2}$ and vice versa, then these accordions are said to be mutually order preserving while $\ell_{1}, \ell_{2}$ are said to have mutually order preserving accordions.

Mutually order preserving accordions are not gaps of a single sibling invariant geolamination as convex hulls of their forward images may have intersecting interiors. Still, their dynamics resembles that of gaps of sibling invariant geolaminations, and so leaves from mutually order preserving accordions have a rigid structure. This is supported by the findings made in Section 3 of [BOPT14]; some of the main results from there are stated here.

Lemma 1.26 (Lemma 3.7 [BOPT14]). If $\ell_{a}=\overline{a b}$ and $\ell_{x}=\overline{x y}, a<x<$ $b<y$, are linked leaves with mutually order preserving accordions and $a, b$ are of period $k$, then $x, y$ are also of period $k$.

To state the main result of Section 3 of [BOPT14] we need Definition 1.27.

Definition 1.27. Let $\ell_{a}=\overline{a b}, \ell_{x}=\overline{x y}$ be linked chords. Set $B=\operatorname{CH}\left(\ell_{a}, \ell_{x}\right)$. Say that the $\sigma_{d}$-dynamics of $B$ is orderly if the following holds.

(1) The order of vertices of $B$ is preserved under iterations of $\sigma_{d}$.

(2) One of the following holds. 
(a) All images of $B$ are pairwise disjoint.

(b) There exist the minimal $r, m$ and $n$ with $\sigma_{d}^{r}(B) \cap \sigma_{d}^{r+m}(B) \neq \emptyset$ and $\sigma_{d}^{r+m n}(B)=B$, and if for any $i \geq 0$ we set $\sigma_{d}^{r+i m}(a)=$ $a_{i}, \sigma_{d}^{r+i m}(b)=b_{i}, \sigma_{d}^{r+i m}(x)=x_{i}, \sigma_{d}^{i+r m}(y)=y_{i}$, then either

$$
a_{0}<x_{0}<b_{0} \leq a_{1}<y_{0} \leq x_{1}<b_{1} \leq a_{2}<y_{1} \leq x_{2}<b_{2} \leq \ldots,
$$

or

$$
x_{0}<a_{0}<y_{0} \leq x_{1}<b_{0} \leq a_{1}<y_{1} \leq x_{2}<b_{1} \leq a_{2}<y_{2} \leq \ldots,
$$

and the set $\bigcup_{i=0}^{n-1} \sigma_{d}^{r+i m} B$ is a component of the orbit of $B$.

(c) The leaves $\ell_{a}, \ell_{x}$ are (pre)periodic of the same eventual period of endpoints.

Despite its appearance, the order among vertices of $B$ and some of its images given in Definition 1.27 can be easily described.

Definition 1.28. If there are chords $\overline{a_{0} b_{0}}, \overline{a_{1} b_{1}}, \ldots, \overline{a_{n} b_{n}}$ with $a_{0} \leq a_{1} \leq$ $\cdots \leq a_{n} \leq a_{0}$ and $b_{0} \leq b_{1} \leq \cdots \leq b_{n} \leq b_{0}$, we say that these chords are positively ordered and denote it by $\overline{a_{0} b_{0}} \leq \overline{a_{1} b_{1}} \cdots \leq \overline{a_{n} b_{n}} \leq \overline{a_{0} b_{0}}$.

Here is how one can restate the long inequalities from Definition 1.27 . Set $\sigma_{d}^{r+i m}\left(\ell_{a}\right)=\ell_{a}^{i}, \sigma_{d}^{r+i m}\left(\ell_{x}\right)=\ell_{x}^{i}$. Then

$$
\ell_{a}^{0} \leq \ell_{x}^{0} \leq \ell_{a}^{1} \leq \ell_{x}^{1} \leq \ldots \ell_{a}^{n-1} \leq \ell_{x}^{n-1} \leq \ell_{a}^{n}=\ell_{a}^{0} \leq \ell_{x}^{n}=\ell_{x}^{0}
$$

or

$$
\ell_{x}^{0} \leq \ell_{a}^{0} \leq \ell_{x}^{1} \leq \ell_{a}^{1} \leq \ldots \ell_{x}^{n-1} \leq \ell_{a}^{n-1} \leq \ell_{x}^{n}=\ell_{x}^{0} \leq \ell_{a}^{n}=\ell_{a}^{0} .
$$

In addition, only consecutive images of $\ell_{a}\left(\ell_{x}\right)$ in this collection can intersect and at most at their endpoints.

The following is the main result of Section 3 of [BOPT14]

Theorem 1.29 (Theorem 3.12 [BOPT14]). Consider linked chords $\ell_{a}, \ell_{x}$ with mutually order preserving accordions, and set $B=\mathrm{CH}\left(\ell_{a}, \ell_{x}\right)$. Then the $\sigma_{d^{-}}$dynamics of $B$ is orderly.

In the rest of this subsection we assume that $\mathcal{L}_{1}, \mathcal{L}_{2}$ are linked cubic geolaminations. Linked leaves of $\mathcal{L}_{1}, \mathcal{L}_{2}$ will often have images which are linked and have mutually order preserving accordions. The only alternative is that they eventually map to leaves which are not linked but share an endpoint. We call this behavior collapsing (around a chain of spikes).

Definition 1.30. Non-disjoint leaves $\ell_{1} \neq \ell_{2}$ are said to collapse around chains of spikes if there are two chains of spikes (one chain in each of our geolaminations) connecting the same two adjacent endpoints of $\ell_{1}, \ell_{2}$. 


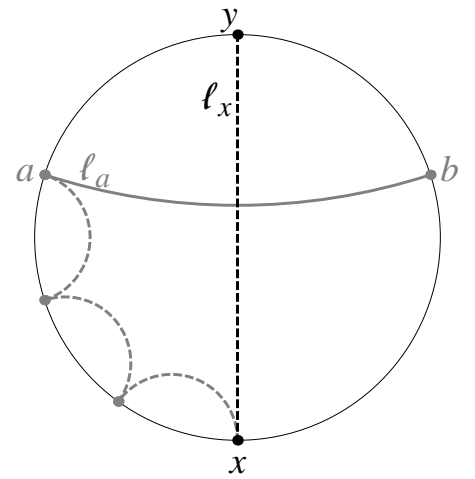

FIGURE 2. This figure illustrates Definition 1.30. Here the leaves $\ell_{a}, \ell_{x}$ collapse around a chain of spikes shown as dashed grey geodesics.

We now list a few results of [BOPT14] on accordions of leaves of $\mathcal{L}_{1}\left(\mathcal{L}_{2}\right)$. Observe that the results obtained in [BOPT14] hold in the degree $d$ case (i.e., for $\sigma_{d}$ ); however, in this paper we confine ourselves to the case $d=3$ (in particular, we define the linkage between geolaminations only for cubic geolaminations) which is why we state results from [BOPT14] in the cubic case only. If $X \subset \mathbb{S}$ is a set of points such that $\left.\sigma_{3}\right|_{X}$ preserves orientation except that some points may be mapped to one, we say that $\left.\sigma_{3}\right|_{X}$ weakly preserves orientation.

Lemma 1.31 (Corollary $4.2[$ [BOPT14] $)$. Let $\ell_{1} \in \mathcal{L}_{1}, \ell_{2} \in \mathcal{L}_{2}$; then for all $k \geq 0$, the restriction of $\sigma_{d}$ to $A_{\ell_{2}}\left(\ell_{1}\right) \cap \mathbb{S}$ is (non-strictly) order preserving. Moreover, if $\ell_{1}$ and $\ell_{2}$ are linked leaves or share an endpoint then the order among their endpoints is weakly preserved under $\sigma_{3}$ unless the common endpoint of $\ell_{1}, \ell_{2}$ is a common vertex of associated critical quadrilaterals of our geolaminations.

Corollary 4.2 in [BOPT14] contains much more detail than Lemma 1.31 . It serves as a major tool as it shows that even though geolaminations $\mathcal{L}_{1}, \mathcal{L}_{2}$ are distinct, crossing leaves of those geolaminations form a set which behaves more or less like a gap of one geolamination.

Lemma 1.32 (Lemma 4.3 [BOPT14]). Suppose that:

(1) $\ell_{1}=\overline{a_{1} b_{1}} \in \mathcal{L}_{1}, \ell_{2}=\overline{a_{1} b_{2}} \in \mathcal{L}_{2}$ are linked leaves or share an endpoint (in the latter case the shared endpoint is not a common vertex of two associated generalized quadrilaterals);

(2) at least of these leaves is not critical;

(3) the order among the endpoints of these leaves is weakly preserved so that, say, $a_{1} \neq a_{2}$ while $\sigma_{3}\left(a_{1}\right)=\sigma_{3}\left(a_{2}\right)$. 
Then there are chains of spikes of $\mathcal{L}_{1}$ and of $\mathcal{L}_{2}$ connecting $a_{1}$ and $a_{2}$ with endpoints in the arc with endpoints $a_{1}, a_{2}$ not containing points $b_{1}, b_{2}$.

The main problem with applications of Lemma 1.31 is that the order among the endpoints of linked leaves of geolaminations $\mathcal{L}_{1}, \mathcal{L}_{2}$ is only weakly preserved. In other words, endpoints of these leaves may collide. Lemma 1.32 describes how this can happen. and shows that if two linked leaves $\ell_{1}, \ell_{2}$ of linked geolaminations have colliding endpoints then these endpoints are connected with specifically located chains of spikes. This allows us to apply Corollary 1.18 and prove Proposition 1.33 which shows that the colliding endpoints are in fact endpoints of specifically located sibling leaves of $\ell_{1}, \ell_{2}$ respectively.

Proposition 1.33. Suppose that $\mathcal{L}_{1}, \mathcal{L}_{2}$ are linked geolaminations and the leaves $\overline{a b} \in \mathcal{L}_{1}$ and $\overline{x y} \in \mathcal{L}_{2}$ are non-critical, linked, and such that a< $x<b<y<a$ and $\sigma_{3}(a)=\sigma_{3}(y)$. Then there exists a leaf $\overline{a x^{\prime}}$ which is a sibling of $\overline{x y}$ such that $x^{\prime}<a<x<y<x^{\prime}$.

Proof. Consider a disjoint sibling collection $\mathcal{A}$ of the leaf $\overline{x y}$ (formed by leaves of $\mathcal{L}_{2}$ ). By Lemma 1.32 there is a chain of spikes of $\mathcal{L}_{2}$ from $a$ to $y$ with endpoints contained in $[y, a]$. Choose the leaf $\overline{a x^{\prime}} \in \mathcal{A}$; then by Corollary $1.18 x^{\prime}<a<x<y<x^{\prime}$.

Finally, we state here Lemma 4.5 from [BOPT14].

Lemma 1.34 (Lemma 4.5 [BOPT14]). If $\ell_{1} \in \mathcal{L}_{1}, \ell_{2} \in \mathcal{L}_{2}$ are linked leaves or share an endpoint and $\sigma_{d}^{t}\left(\ell^{1}\right)$ and $\sigma_{d}^{t}\left(\ell^{2}\right)$ do not collapse around chains of critical chords for any $t$, then there exists an $N$ such that the $\sigma_{d}^{N}$-images of $\ell^{1}, \ell^{2}$ are linked and have mutually order preserving accordions. Thus, if $\bar{m}_{1} \in \mathcal{L}_{1}, \bar{m}_{2} \in \mathcal{L}_{2}$ are non-disjoint leaves then for any $n$ the leaves $\sigma_{3}^{n}\left(\bar{m}_{1}\right), \sigma_{d}^{n}\left(\bar{m}_{2}\right)$ are non-disjoint.

1.3. Proper Geolaminations. Given a lamination $\sim$, the generated geolamination $\mathcal{L}_{\sim}$ is formed by the edges of $\sim$-classes (see Definition 1.3); if $\sim$ is invariant, the family of all such geolaminations is denoted $\mathbb{L} \mathcal{Q}$. While $\mathbb{L} \mathcal{Q}$-geolaminations are natural objects, they are often difficult to work with. To overcome these difficulties we define a wider and often more convenient class of geolaminations. This will also be helpful in dealing with geolaminations in the parameter space.

1.3.1. Basic definitions and properties. First we introduce an inverse process to that from Definition 1.3 .

Definition 1.35. Suppose that a family $\mathcal{F}$ of chords in $\overline{\mathbb{D}}$ is given. Define an equivalence relation $\sim_{\mathcal{F}}$ as follows: $x \sim_{\mathcal{F}} y$ if and only if there exists a finite concatenation of chords of $\mathcal{F}$ joining $x$ and $y$. We say that $\mathcal{F}$ generates (or gives rise to) the equivalence relation $\sim_{\mathcal{F}}$. 
Clearly, $\sim_{\mathcal{F}}$ is an equivalence relation for any collection of chords $\mathcal{F}$ $(\mathcal{F}$ does not have to be invariant or even closed). We are interested in families $\mathcal{F}$ such that the equivalence relation $\sim_{\mathcal{F}}$ is a lamination with the specific properties from Definition 1.1 (in the non-invariant case) and, in addition, from Definition 1.2 (in the invariant case). Observe that there are invariant geolaminations not from $\mathbb{L} \mathcal{Q}$ which generate invariant laminations. For example, inserting a leaf into the interior of a finite gap $G$ of an $\mathbb{L} \mathcal{Q}$-geolamination and then inserting leaves from its grand orbit into appropriate sets from the grand orbit of $G$ does not change the underlying equivalence relation. Whether this can be done depends on the dynamics of $G$ (e.g., it can always be done if $G$ is critical or wandering), but the resulting geolamination is no longer in $\mathbb{L} \mathcal{Q}$.

Definition 1.36. A family of chords $\mathcal{F}$ such that the generated equivalence relation $\sim_{\mathcal{L}}$ is a lamination is said to be proper. Denote by $\mathbb{L} \mathbb{P}$ the family of all invariant proper geolaminations and by $\mathbb{L} \mathbb{P}_{d}$ the set of $\sigma_{d}$-invariant geolaminations from $\mathbb{L} \mathbb{P}$.

The following lemma can be found in [BMOV13].

Lemma 1.37. Suppose that a $\sigma_{d}$-invariant geolamination $\mathcal{L}$ is proper (i.e. generates a lamination $\sim_{\mathcal{L}}$ ). Then $\sim_{\mathcal{L}}$ is a $\sigma_{d}$-invariant lamination.

There are useful criteria for an invariant geolamination to be proper.

Definition 1.38. Two non-degenerate leaves with common endpoint $v$ and the same non-degenerate image are said to form a critical wedge (the point $v$ is said to be its vertex). Call a non-degenerate leaf improper if it has exactly one periodic endpoint.

The dynamics of improper leaves is described in Lemma 1.39

Lemma 1.39. Let $\ell=\overline{p x}$ be an improper leaf with $p$ periodic of period $k$ and $x$ non-periodic. Then it has an eventual image-leaf which is either a critical leaf with a periodic endpoint or a leaf with exactly one periodic endpoint which maps to a periodic leaf (in the latter case this image-leaf and the appropriate image of it form a critical wedge).

Proof. Consider the leaves $\sigma_{d}^{k n}(\ell)$. Suppose that all points $p, x, \sigma_{d}^{k}(x)$, $\sigma_{d}^{2 k}(x), \ldots$ are distinct. Then by Lemma 1.11 applied to $\sigma_{d}^{k}$, we may assume that $p<x<\sigma_{d}^{k}(x)<\sigma_{d}^{2 k}(x)<\ldots$ Since $\sigma_{d}$ is expanding, this is impossible. Hence there exists a minimal $N$ such that either $\sigma_{d}^{N k}(x)=p$ or (again by Lemma 1.11) $\sigma_{d}^{N k}(x)$ is a $\sigma_{d}^{k}$-fixed point distinct from $p$. In the first case there exists $0 \leq i<k$ such that $\sigma_{d}^{k(N-1)+i}(\ell)$ is a critical leaf with a periodic endpoint and in the second case there exists $0 \leq i<k$ such that $\sigma_{d}^{k(N-1)+i}(\ell) \cup \sigma_{d}^{k(N-1)+i+1}$ is a critical wedge with a periodic edge. 
Lemma 1.40 presents the above mentioned criteria for an invariant geolamination to be proper.

Lemma 1.40 (cf [BMOV13]). The following properties are equivalent.

(1) The $\sigma_{d}$-invariant geolamination $\mathcal{L}$ is proper (i.e., $\sim_{\mathcal{L}}$ is a lamination).

(2) The $\sigma_{d}$-invariant geolamination $\mathcal{L}$ has no critical leaves (wedges) with a periodic endpoint (vertex).

(3) The $\sigma_{d}$-invariant geolamination $\mathcal{L}$ has no improper leaves.

(4) The $\sigma_{d}$-invariant geolamination has neither a critical leaf with a periodic endpoint nor a critical wedge with a periodic leaf.

In particular, concatenations of leaves of $\mathcal{L}$ are finite.

Proof. Parts (1) - (3) of the lemma are equivalent by [BMOV13]. Clearly, (3) implies (4). The opposite direction follows from Lemma 1.39.

Consider the difference between a proper geolamination $\mathcal{L}$ and the $\mathbb{L} \mathcal{Q}$ geolamination $\mathcal{L}_{\sim_{\mathcal{L}}}$ generated by the lamination $\sim_{\mathcal{L}}$.

Definition 1.41. Let $\mathcal{L}$ be a proper geolamination. For a $\sim_{\mathcal{L}}$-class $\mathfrak{g}$, denote by $\mathfrak{g}_{\mathcal{L}}$ the collection of all leaves of $\mathcal{L}$ connecting points of $\mathfrak{g}$.

Lemma 1.42 easily follows from the definitions.

Lemma 1.42. Let $\mathcal{L}$ be a proper geolamination and let $\mathfrak{g}_{\mathcal{L}}$ be a $\sim_{\mathcal{L}}$-class. Then all leaves of $\mathcal{L}$ intersecting $\mathfrak{g}_{\mathcal{L}}$ are disjoint from all other leaves of $\mathcal{L}$; for any points $a, b \in \mathfrak{g}_{\mathcal{L}}$ there is a finite chain of leaves from $\mathfrak{g}_{\mathcal{L}}$ connecting $a$ and $b$.

For a $\sigma_{d}$-invariant geolamination, by Theorem 1.10 and Definition 1.7 for any gap $G$ the map $\left.\sigma_{d}\right|_{\operatorname{Bd}(G)}$ is the composition of a monotone map and a covering map of some degree. If $G$ is of period $k$, then the same holds for $\sigma_{d}^{k}: \operatorname{Bd}(G) \rightarrow \operatorname{Bd}(G)$; the degree of the corresponding map is called the degree of $G$. A concatenation of chords is trivial if it consists of one chord.

Consider a lamination $\sim$. By [BL02] for any infinite gap $G$ of $\sim$ the set $G \cap \mathbb{S}$ is a Cantor set (thus, by Definition $1.5 G$ is a Fatou gap). Recall, that $\psi_{G}: \operatorname{Bd}(G) \rightarrow \mathbb{S}$ is the monotone map which collapses all edges of $G$ to points (since $G \cap \mathbb{S}$ is a Cantor set, there are no non-trivial concatenations of edges of $G$ ). By [BL02], a periodic infinite gap $G$ of $\sim$ of period $k$ can be of one of two types. If $G$ is of degree 1 we associate to it its irrational rotation number $\rho$, and $\left.\sigma_{d}^{k}\right|_{\operatorname{Bd}(G)}$ is semiconjugate by $\psi_{G}$ to the circle rotation by the angle $\rho$. Such gaps are called Siegel gaps. Now, $\left.\sigma_{d}^{k}\right|_{\operatorname{Bd}(G)}$ can be of degree $r>1$. Then by [BL02] the map $\psi_{G}$ semiconjugates $\left.\sigma_{d}^{k}\right|_{\operatorname{Bd}(G)}$ and $\left.\sigma_{r}\right|_{\mathbb{S}}$. Such gaps $G$ are called Fatou gaps of degree $r$. Observe finally that by [BL02] any infinite gap $G$ of $\sim$ is (pre)periodic. 
Now, if $\mathcal{L}$ is a proper geolamination, we can construct the lamination $\sim_{\mathcal{L}}$, the geolamination $\mathcal{L}_{\sim_{\mathcal{L}}}$, and apply to $\sim_{\mathcal{L}}$ and $\mathcal{L}_{\sim_{\mathcal{L}}}$ the results of [BL02] quoted in the previous paragraph together with Lemma 1.42. This gives the following description of infinite gaps of proper geolaminations. A periodic gap of period $k$ is said to be identity return (under $\sigma_{d}^{k}$ ) if $\sigma_{d}^{k}$ fixes its vertices.

Lemma 1.43. Any infinite gap $G$ of a proper geolamination $\mathcal{L}$ is a (pre)periodic Fatou gap. If $G$ is a k-periodic gap of $\mathcal{L}$ of degree $r$, then the following holds.

(1) If $r=1$, then the map $\psi_{G}$ semiconjugates $\left.\sigma_{d}^{k}\right|_{\operatorname{Bd}(G)}$ and the rotation of $\mathbb{S}$ by an irrational number $\rho_{G}$.

(2) If $r>1$, then the map $\psi_{G}$ semiconjugates $\left.\sigma_{d}^{k}\right|_{\operatorname{Bd}(G)}$ and $\sigma_{r}$. If a maximal concatenation $L$ of periodic leaves on the boundary of $G$ is non-trivial and $\sigma_{d}^{k n}(L) \cap L \neq \emptyset$, then its convex hull $H$ is a periodic identity return gap under $\sigma^{k n}$.

Proof. We only need to prove the last claim from part (2). Suppose that $L=\ell_{1} \cup \cdots \cup \ell_{m}$ is a maximal concatenation (in this order) of periodic leaves on the boundary of $G$ and $m>1$. Then the convex hull $H$ of $L$ can be obtained by connecting the appropriate endpoints of $\ell_{1}$ and $\ell_{m}$ with a chord which we denote by $\bar{n}$. If $k n$ is the period of all the leaves in $L$ then $\sigma_{d}^{k n}(H)=H$ and $\sigma_{d}^{k}$ fixes all the vertices of $H$ (observe that by Lemma 1.26 all leaves in $L$ have the same period). Suppose that there exists a number $s<k$ such that $\sigma_{d}^{s}(H)=H$. Then leaves in $L$ are not fixed under $\sigma_{d}^{s}$ and one of them will have to be mapped onto $\bar{n}$, a contradiction.

Definition 1.44. Periodic gaps of degree $r$ of proper geolaminations are said to be either Siegel gaps (if $r=1$ ) or Fatou gaps of degree $r$ (if $r>1$ ). For any gap $G$ of period $k$, a $k$-periodic edge of $G$ is said to be refixed; $\left.\sigma_{d}^{k}\right|_{\operatorname{Bd}(G)}$ is called a remap.

Let $\mathcal{N} \mathcal{P}$ be the set of all critical leaves with non-periodic endpoints.

Lemma 1.45. Let $\mathcal{L}$ be a proper geolamination with a critical leaf $D \in$ $\mathcal{N P}$. Let $A$ be the convex hull of the $\sim_{\mathcal{L}}$-class containing the endpoints of $D$. Then $A$ is a leaf or a finite gap and the following cases are possible.

(1) The set $A$ is critical such that $\left.\sigma_{3}\right|_{A}$ is 3-to-1. Moreover, either $A$ is an all-critical triangle, or there is a critical gap or leaf $C \subset$ $A, C \neq D$ of $\mathcal{L}$ such that $\left.\sigma_{3}\right|_{C}$ is of degree 2.

(2) Otherwise there is a critical set $C$ that is either an infinite gap or the convex hull of $a \sim_{\mathcal{L}^{-}}$class such that $\left.\sigma_{3}\right|_{C}$ is of degree two and one of the following holds.

(a) $C \cap \mathbb{S}$ is finite, $C$ is disjoint from $A$, and $\left.\sigma_{3}\right|_{C}$ is two-to-one.

(b) $C$ is a periodic Fatou gap. 
(c) $C$ is a preperiodic infinite gap which eventually maps to a periodic Siegel gap $U$ with $D$ being an edge of $U$.

Proof. Since $\sim_{\mathcal{L}}$ is a lamination, then $A$ is either a leaf (then it coincides with $D$ ), or a finite critical gap. If $A$ is of degree 3 then it is the unique critical set of $\sim_{\mathcal{L}}$ because we are considering the cubic case. The rest of case (1) is left to the reader (recall that the degree of a gap is defined in Definition 1.8).

Now, suppose that $A$ is of degree 2 and denote the second critical set of $\sim_{\mathcal{L}}$ by $C$. If $C \cap \mathbb{S}$ is finite then (a) holds. Thus we may assume that $C$ is an infinite gap. If $C$ is periodic, then by Lemma 1.43 (b) holds. If $C$ is preperiodic then an eventual image of $C$ is a periodic gap $U$ of degree 1 (it cannot be of degree 2 because we work with the cubic case and there is not enough criticality). By Lemma $1.43 U$ is a Siegel gap. It is well-known that in any cycle of Siegel gaps there must be a gap with at least one critical edge (see, e.g., Lemma 2.13 [BOPT14]). Hence we may assume that $U$ has $D$ as its critical edge (under the assumptions $D$ is the unique critical leaf of $\mathcal{L}$ as otherwise $C$ would have been another critical edge of $U$ while we assume that $C$ is an infinite gap).

Proper geolaminations described in case (2)(c) of Lemma 1.45 are said to be Siegel geolaminations of capture type.

1.3.2. Finding proper sub-geolaminations. By Theorem 1.10 the space $\mathbb{L}_{d}$ of all $\sigma_{d}$-invariant sibling geolaminations is compact. This allows one to assign geolamination(s) to every polynomial approximated by polynomials with locally connected Julia sets: if $P_{i} \rightarrow P$ and $P_{i}$ 's are polynomials with locally connected Julia sets and geolaminations $\mathcal{L}_{P_{i}}$, then any limit geolamination $\lim _{i \rightarrow \infty} \mathcal{L}_{P_{i}}$ can be associated to $P$. However, while $\mathcal{L}_{P_{i}}$ 's are proper, their limit geolaminations might be not proper because the set of proper geolaminations $\mathbb{L} \mathbb{P}_{d} \subset \mathbb{L}_{d}$ is not closed. To overcome this difficulty, we will develop techniques to associate a proper geolamination to many geolaminations of $\mathbb{L}_{3}$. A fact which plays a central role in this procedure is that improper leaves are isolated. Say that a leaf $\ell$ of a geolamination $\mathcal{L}$ is a limit leaf if it is the limit of a sequence of leaves of $\mathcal{L}$ distinct from $\ell$ itself.

Recall (Definition 1.38) that a non-degenerate leaf is improper if it has exactly one periodic endpoint. Also, given an arc $I \subset \mathbb{S}$ set $|I|$ to be its length.

Proposition 1.46. Let $\mathcal{L} \in \mathbb{L}_{d}$ be a $\sigma_{d}$-invariant sibling geolamination. Then every improper leaf of $\mathcal{L}$ is isolated in $\mathcal{L}$.

Proof. Let $\ell$ be an improper leaf of $\mathcal{L}$. By Lemma 1.39, an eventual image $\sigma_{d}^{m}(\ell)=\overline{p x}$ of $\ell$ is either (1) a critical leaf with one periodic endpoint $p$ 
of period $k$ or (2) a leaf with exactly one periodic endpoint of period $k$ whose $\sigma_{d}^{k}$-image $\sigma^{k}\left(\sigma_{d}^{m}(\ell)\right)$ is a $\sigma_{d}^{k}$-periodic leaf $\left(\sigma_{d}^{m}(\ell)\right.$ and $\sigma_{d}^{m+k}(\ell)$ form a critical wedge for $\left.\sigma_{d}^{k}\right)$. We show by way of contradiction that $\sigma_{d}^{m}(\ell)=\overline{p x}$ is isolated in either case.

(1) In this case $\sigma_{d}^{k}(x)=p$. Since $\sigma_{d}$ is locally order preserving then for any chord $\overline{p^{\prime} x^{\prime}}$ close to but disjoint from $\overline{p x}$ its $\sigma_{d}^{k}$-image crosses $\overline{p x}$. Hence $\overline{p x}$ is a limit of leaves which meet $\overline{p x}$. Since $\sigma_{d}$ is expanding, then the $\sigma_{d}^{k}$ image of a chord $\overline{x p^{\prime}}$ close to $\overline{p x}$ crosses itself. On the other hand, let $\overline{p x^{\prime}}$ be a leaf of $\mathcal{L}$, and assume (without loss of generality) that $p<x^{\prime}<x$ and that $x^{\prime}$ is close to $x$. Set $\sigma_{d}^{k}\left(\overline{p x^{\prime}}\right)=\overline{p y}$; clearly, $x<y<p$. Then by Lemma 1.17 there exists a sibling leaf $\overline{x z}$ of $\overline{p y}$ emanating from $x$ and such that $p<z<x$. Since $x^{\prime}$ is close to $x$ we may assume that $[y, p]=\sigma_{d}^{k}\left[x^{\prime}, x\right]$. Since $\sigma_{d}^{k}$ is expanding, $[y, p]=d \cdot\left[x^{\prime}, x\right]>\left|\left[x^{\prime}, x\right]\right|$. On the other hand, the fact that $\overline{p y}$ and $\overline{x z}$ are siblings and the fact that $[y, p]$ is a small arc (because $x^{\prime}$ and $x$ are close) imply that the $|[z, x]| \geq|[y, p]|$. Thus, $|[z, x]|>\left|\left[x^{\prime}, x\right]\right|$. Hence, $\overline{z x}$ crosses $\overline{p x^{\prime}}$, a contradiction.

(2) Let $\sigma_{d}^{m}(\ell)=\overline{p x}, \sigma_{d}^{m+k}(\ell)=\overline{p x^{\prime}}, \sigma_{d}^{k}(x)=x^{\prime}<p<x$ (thus, $\overline{p x} \cup \overline{p x^{\prime}}$ is a critical wedge). Then by Corollary 1.12 so there are at most finitely many leaves separating $\overline{p x}$ and $\overline{p x^{\prime}}$. This implies that if the leaf $\overline{p x}$ is a limit leaf then there exists a sequence of leaves $\overline{q t}$ which converge to $\overline{p x}$ and are such that $p \leq q<t \leq x$. Since $\sigma_{d}$ is expanding, if $p<q$ then the $\sigma_{d}^{k}$-image of $\overline{q t}$ crosses $\overline{p x}$. Thus, $p=q$. Then, by continuity infinitely many leaves $\sigma_{d}^{k}(\overline{q t})$ separate $\overline{p x}$ and $\overline{p x^{\prime}}$, a contradiction with the above.

Call a disjoint sibling collection improper if it includes an improper leaf.

Lemma 1.47. If a leaf $\ell$ is periodic or has non-preperiodic endpoints, then no disjoint sibling collection of $\ell$ is improper.

Proof. The case when the endpoints of $\ell$ are non-preperiodic is obvious. If $\ell=\overline{x y}$ is periodic and $\ell^{\prime}$ is a disjoint sibling of $\ell$ then both endpoints of $\ell^{\prime}$ are non-periodic as desired.

We need the following definition.

Definition 1.48. Let $\mathcal{L} \in \mathbb{L}_{d} \backslash \mathbb{L} \mathbb{P}_{d}$. Let $\mathcal{L}^{p} \subset \mathcal{L}$ be the set of proper leaves $\ell \in \mathcal{L}$ such that they and all their (eventual) non-critical images have disjoint sibling collections consisting of proper leaves.

Arguments similar to those used in the proof of Theorem 1.15 allow us to find proper sub-geolaminations.

Lemma 1.49. Let $\mathcal{L} \in \mathbb{L}_{d} \backslash \mathbb{L} \mathbb{P}_{d}$. Then $\mathcal{L}^{p}$ is a proper $\sigma_{d}$-invariant sibling geolamination containing all periodic leaves of $\mathcal{L}$, all leaves of $\mathcal{L}$ with non-preperiodic endpoints, and all critical leaves of $\ell$ with non-periodic endpoints. 
Proof. Let $\ell \in \mathcal{L}^{p}$. Then $\ell$ is proper. By definition, $\sigma_{d}(\ell) \in \mathcal{L}^{p}$. Moreover, by definition $\ell$ has a disjoint sibling collection consisting of proper leaves all of which also belong to $\mathcal{L}^{p}$. Now consider pullbacks of $\ell$ and show that among them we can choose a disjoint sibling collection consisting of proper leaves. Indeed, the only way $\ell$ can have an improper pullback $\ell^{\prime}$ is when $\ell$ is periodic while $\ell^{\prime}$ has exactly one periodic endpoint. To handle this case, notice that $\ell$ must also have a purely periodic pullback (e.g., if $\ell$ is of period $s$ we can always choose $\sigma_{d}^{s-1}(\ell)$ as such a leaf). Choosing this pullback and its disjoint sibling collection we see that this entire collection consists of proper leaves as desired.

By definition, it remains to show that $\mathcal{L}^{p}$ is closed. To this end we need to check if all removed leaves are isolated. Indeed, a leaf $\ell$ is removed if for some $i \geq 0$ all disjoint sibling collections of $\sigma_{d}^{i}(\ell)$ include an improper leaf (which is isolated by Proposition 1.46). By Lemma 1.14 this implies that $\sigma_{d}^{i}(\ell)$ is isolated. Indeed, if $\sigma_{d}^{i}(\ell)$ is non-isolated, then by Lemma 1.14 it has a disjoint sibling collection consisting of non-isolated leaves which (by Proposition 1.46) are all proper, a contradiction. Hence $\sigma_{d}^{i}(\ell)$ is isolated as desired. The last claim of the lemma follows from Lemma 1.47 and the definition of $\mathcal{L}^{p}$.

Proposition 1.50 provides conditions whereby (the grand orbit of) a periodic leaf can be added to a proper geolamination $\mathcal{L}$ to create another proper geolamination.

Proposition 1.50. Let $\mathcal{L}$ be a proper geolamination and $L$ be a cycle of periodic leaves which do not cross leaves of $\mathcal{L}$. Then there exists a proper geolamination $\widehat{\mathcal{L}}$ containing $L \cup \mathcal{L}$ such that $\widehat{\mathcal{L}} \backslash \mathcal{L}$ consists of leaves which either eventually map to $L$ or are limits of such leaves.

Proof. The proof is rather straightforward, so we only sketch it. To construct $\widehat{\mathcal{L}}$, we need to pull back $L$ in a step by step fashion so that on each step disjoint sibling collections are formed. This is immediate if a leaf which is being pulled back is not contained in a gap which itself is the image of a critical set. Otherwise it suffices to choose the pullbacks of the leaf in question inside of the appropriate critical sets so that again we will be getting one or several disjoint sibling collections. Repeating this countably many times we will make the first step in the construction. It is easy to see that when we take the closure, the resulting geolamination is a $\sigma_{d}$-invariant sibling geolamination [BMOV13]. It follows from Proposition 1.46 that $\widehat{\mathcal{L}}$ is a proper geolamination.

\section{MAin Results}

Consider cubic (geo)laminations. We need the following definition. 
Definition 2.1. Two generalized critical quadrilaterals form an admissible (cubic) qc-portrait if (1) if a generalized critical quadrilateral has a periodic vertex and a non-degenerate image then it must have a periodic edge, and (2) the elements of the qc-portrait and all their images intersect at most over a common edge or vertex. By Theorem 1.19, the sets of the orbits of elements of an admissible qc-portrait QCP can be pulled back to form a $\sigma_{3}$-invariant geolamination; any such geolamination is denoted by $\mathcal{L}_{\mathrm{QCP}}$.

Recall that $\mathcal{N P}$ is the set of critical leaves with non-periodic endpoints.

Definition 2.2. Let $\mathbb{L} \mathbb{P}_{3}^{n p}$ be the set of all proper geolaminations with a critical leaf from $\mathcal{N P}$ except for Siegel geolaminations of capture type. Let $\mathcal{Q C} \mathcal{P}_{3}^{n p}$ be the collection of all admissible qc-portraits with a critical leaf from $\mathcal{N} \mathcal{P}$ as the second element. For $D \in \mathcal{N} \mathcal{P}, \mathcal{S}_{D}$ is the collection of admissible qc-portraits with $D$ as the second element.

Recall that the sets $\mathbb{L} \mathbb{P}_{3}^{n p}, \mathcal{Q C P}{ }_{3}^{n p}$ and $\mathcal{S}_{D}$ are endowed with the Hausdorff metric and the induced topology. From now on whenever we talk about a privileged qc-portrait of a geolamination $\mathcal{L} \in \mathbb{L P}_{3}^{n p}$ (the notion of a privileged qc-portrait can be found in the Introduction and is formally given in Definition 3.1) we always assume that its second element is a critical leaf with non-periodic endpoints.

Theorem A. Each $\mathcal{L} \in \mathbb{L} \mathbb{P}_{3}^{n p}$ has at least one and no more than finitely many privileged qc-portraits. For every $\mathrm{QCP} \in \mathcal{Q C P}{ }_{3}^{n p}$, there exists $\mathcal{L} \in$ $\mathbb{L P}_{3}^{n p}$ such that $\mathrm{QCP}$ is privileged for $\mathcal{L}$. Moreover, $\mathcal{S}_{D}$ is compact.

It turns out that privileged portraits properly capture dynamics.

Theorem B. Suppose that $\mathrm{QCP}_{1}=\left(Q_{1}, D\right), \mathrm{QCP}_{2}=\left(Q_{2}, D\right)$ are privileged qc-portraits for geolaminations $\mathcal{L}_{1}, \mathcal{L}_{2} \in \mathbb{L} \mathbb{P}_{3}^{n p}$ such that $\sigma_{3}\left(Q_{1}\right) \cap$ $\sigma_{3}\left(Q_{2}\right) \neq \emptyset$. Then $\sim_{\mathcal{L}_{1}}=\sim_{\mathcal{L}_{2}}$.

Observe that if $\mathrm{QCP}_{1}, \mathrm{QCP}_{2}$ are linked privileged qc-portraits for geolaminations $\mathcal{L}_{1}, \mathcal{L}_{2} \in \mathbb{L} \mathbb{P}_{3}^{n p}$ then there exists a critical leaf $D \in \mathcal{N} \mathcal{P}$ such that $\mathrm{QCP}_{1}, \mathrm{QCP}_{2} \in \mathcal{S}_{D}$.

In contrast to the quadratic case, where there are no linked minors, there may be linked qc-portraits in $\mathcal{Q} \mathcal{C} \mathcal{P}_{3}^{n p}$, but the laminations specified by these linked qc-portraits are the same. We use this result to study $\mathcal{S}_{D}$, which is naturally identified with the set of $\mathbb{L} \mathbb{P}_{3}$-geolaminations containing $D$. Each $(Q, D) \in \mathcal{S}_{D}$ is tagged by the chord or point (the minor) $\sigma_{3}(Q)$. Denote by $\mathrm{CML}_{D}$ the family of all such leaves $\sigma_{3}(Q)$. We will show that $\mathrm{CML}_{D}$ is an appropriate cubic analog of Thurston's QML.

Theorem C. The family $\mathrm{CML}_{D}$ is proper, so that the generated equivalence relation $\sim_{\mathrm{CML}_{D}}=\sim_{D}$ is a lamination. Each $\sim_{D}$-class corresponds to 
a unique cubic lamination $\mathcal{L}$. Conversely, every cubic lamination which is not of capture Siegel type and is such that endpoints of $D$ are equivalent corresponds to $a \sim_{D}$-equivalence class.

If $D=\overline{a b}$, then Theorem $\mathrm{C}$ equips the set $\mathrm{LAM}_{D}$ of cubic laminations $\approx$ such that $a \approx b$ and which are not of Siegel capture type with the quotient topology of the unit circle. Also, suppose that a lamination $\approx$ with $a \approx b$ is of Siegel capture type. Then there is only one way this can happen. Namely, $\approx$ must then have a periodic Siegel gap $U$ with $D$ being an edge of $U$ and a non-periodic pullback $V$ of $U$ which maps forward in a two-to-one fashion.

Finally we study the $\sim_{D}$-classes. First we modify the classical notion of the minor of a geolamination in the quadratic case.

Definition 2.3 (Minor sets in the quadratic case [Thu85]). For a quadratic lamination $\sim$, let $C$ be the critical set of $\sim$. If $C$ is finite, let $m_{\sim}=\sigma_{2}(C)$. If $C$ is a Fatou gap of degree two, let $m_{\sim}$ be the $\sigma_{2}$-image of the refixed edge of $C$. The set $m_{\sim}$ is called the minor set of $\sim$.

Note that in the quadratic case minor sets of quadratic laminations coincide with the convex hulls of $\sim_{\mathrm{QML}}$-classes. We want to extend these ideas to the cubic case. In the cubic case, similar to the quadratic case, minor sets can be introduced for all laminations $\sim$ from $\mathrm{LAM}_{D}$ as images of the first critical set of $\sim$ (if it is finite) or the image of the refixed edge of the periodic Fatou gap $U$ of degree two of $\sim$ (if it exists). However in the cubic case there is a new phenomenon which causes these collections of minor sets taken for laminations $\sim$ from $\mathrm{LAM}_{D}$ to be insufficient. We overcome this difficulty by modifying Definition 2.3 below.

Definition 2.4 (Minor sets in the cubic case). If $\sim \in \mathrm{LAM}_{D}$, let $D_{\sim}$ be the $\sim$-class containing the endpoints of $D$. Also, if $\sim$ has a $k$-periodic critical Fatou gap $U$, of degree two, let $M_{\sim}$ be the $\sim$-class of the unique edge of $U$ of period $k$. Let $C_{\sim}$ be either the first critical set of $\sim$ (if it is different from $D_{\sim}$ and finite), $M_{\sim}$ (if the first critical set of $\sim$ is a periodic Fatou gap $U$ of degree two), or $\mathrm{CH}\left(D_{\sim}\right)$ (if $\sim$ has a unique critical class $D_{\sim}$ ). Set $\sigma_{3}\left(C_{\sim}\right)=m_{\sim}$ and call $m_{\sim}$ the minor set of $\sim$.

Now we are ready to state Theorem D.

Theorem D. Classes of $\sim_{D}$ coincide with the minor sets $m_{\sim}$ where $\sim \in$ $\mathrm{LAM}_{D}$.

\section{Privileged QC-Portraits For Proper Geolaminations}

Let us recall the definition of a privileged portrait. 
Definition 3.1. If $\mathcal{L} \in \mathbb{L} \mathbb{P}_{3}^{n p}$ has a critical leaf $D$ with non-periodic endpoints then a qc-portrait $\mathrm{QCP}=(Q, D)$ is called privileged for $\mathcal{L}$ if and only if $Q \subset C$ where $C \neq D$ is a critical set of $\mathcal{L}$ and either $C$ is finite, or $C$ is a periodic Fatou gap of degree two and period $k$ and $Q$ is a collapsing quadrilateral which is a convex hull of a (possibly degenerate) edge $\ell$ of $C$ of period $k$ and another edge $\hat{\ell}$ of $C$ such that $\sigma_{3}(\ell)=\sigma_{3}(\hat{\ell})$.

Observe that edges of the set $Q$ from Definition 3.1 are not necessarily leaves of $\mathcal{L}$. For example, if $Q$ is contained in a periodic Fatou gap of degree two then improper edges of $Q$ are not leaves of the proper geolamination $\mathcal{L}$.

Lemma 3.2 immediately follows from the definitions and Lemma 1.45 and is stated here without proof.

Lemma 3.2. Each $\mathcal{L} \in \mathbb{L} \mathbb{P}_{3}^{n p}$ has at least one and no more than finitely many privileged qc-portraits.

In the rest of this section we show that every $\mathrm{QCP} \in \mathcal{Q C P}_{3}^{n p}$ is a privileged qc-portrait of some $\mathcal{L} \in \mathbb{L} \mathbb{P}_{3}^{n p}$. Recall Definition 2.1.

Definition 3.3. An admissible (cubic) qc-portrait is an ordered pair of generalized critical quadrilaterals $(A, B)$ such that $A \neq B$, and, moreover, $A, B$ and all their images intersect at most over a common edge or vertex, and if $A$ or $B$ has a periodic vertex then it either has a degenerate image or has a periodic edge.

It turns out that if $B \in \mathcal{N} \mathcal{P}$ then it suffices to make sure that the first part of the definition of an admissible qc-portrait holds, the second one then will automatically follow. To prove that, given a qc-portrait QCP one needs to use Theorem 1.19 and construct corresponding (sibling) invariant geolaminations each of which is denoted by $\mathcal{L}_{\mathrm{QCP}}$.

Proposition 3.4. Let $(Q, D)$ be a cubic qc-portrait with $D \in \mathcal{N} \mathcal{P}$ and such that $Q \neq D$ and all their images intersect at most over a common edge or vertex. Moreover, if $Q$ is an all-critical triangle viewed as a generalized quadrilateral then we assume that if $Q$ has a periodic vertex this vertex is considered as an edge of $Q$. Then $(Q, D)$ is admissible. Moreover, if $Q$ has no periodic vertices, then any geolamination containing $\{Q, D\}$ is proper and $\mathrm{QCP}=(Q, D)$ is a privileged qc-portrait for it.

Proof. Let $\mathcal{L}_{\mathrm{QCP}}$ denote a geolamination which contains $(Q, D)$. If $\mathcal{L}_{\mathrm{QCP}}$ is not proper then there is a periodic point $p$ and a critical leaf/wedge $L$ with vertex at $p$. Since the endpoints of $D$ are non-periodic, $p \notin D$. Thus if vertices of $Q$ are non-periodic, then $p \notin Q$ which implies that such a set $L$ does not exist, $\mathcal{L}_{\mathrm{QCP}}$ is proper, and by definition QCP is a privileged qc-portrait for $\mathcal{L}_{\mathrm{QCP}}$. This proves the second claim of the lemma. 
To prove the first claim, assume that $Q$ is a collapsing quadrilateral with a periodic vertex $p$. Clearly, two edges of $Q$ with periodic vertex, say, $p$, form a critical wedge $L$. Moreover, it follows that $\mathcal{L}$ has no critical leaves with a periodic endpoint, and the unique critical wedge of $\mathcal{L}$ is $L$. Since by Lemma $1.39 \mathcal{L}$ has at least one critical wedge with a periodic edge, the second claim of the lemma follows.

Corollary 3.5 immediately follows.

Corollary 3.5. Let $\mathrm{QCP}=(Q, D) \in \mathcal{Q C} \mathcal{P}_{3}^{n p}$. Then $\sigma_{3}^{k}(Q)$ is not an improper leaffor all $k>0$.

Proof. If $Q$ has a periodic vertex, then by Proposition 3.4 it has a periodic edge, and the result is immediate. Otherwise, by Proposition 3.4 any pullback geolamination $\mathcal{L}_{\mathrm{QCP}}$ is proper. Since all $\sigma_{3}$-images of $Q$ are in $\mathcal{L}_{\mathrm{QCP}}$, none of them are improper.

To prove that if $\mathrm{QCP}=(Q, D) \in \mathcal{Q C} \mathcal{P}_{3}^{n p}$ then $\mathrm{QCP}$ is a privileged qcportrait of a proper cubic geolamination we use the following strategy. First we use Theorem 1.19 and construct a pull-back geolamination $\mathcal{L}_{\mathrm{QCP}}$. Then we clean $\mathcal{L}_{\mathrm{QCP}}$ using Lemma 1.49 and obtain a proper geolamination $\mathcal{L}^{p r}$. Now consider two cases. The case when sets from QCP have no periodic vertices is easier to handle. In this case by Proposition 3.4 any pullback geolamination $\mathcal{L}_{\mathrm{QCP}}$ is proper. By definition $\mathcal{L}_{\mathrm{QCP}}$ is not of Siegel capture type. Since $Q$ and $D$ by construction remain critical sets of $\mathcal{L}_{\mathrm{QCP}}, \mathrm{QCP}$ is privileged for $\mathcal{L}_{\mathrm{QCP}}$ by definition.

Consider now the case when $Q$ has a periodic vertex. By Proposition 3.4 then $Q$ is either a critical leaf, or an all-critical triangle, or a collapsing quadrilateral with a periodic edge. To show that $(Q, D)$ is a privileged qcportrait for some proper cubic geolamination we need the following fact.

Proposition 3.6 ([Sch09, Thu85] $)$. Let $\bar{c}$ be a $\sigma_{2}$-critical leaf with a periodic endpoint $p$ of period $k>1$. Then there exists a unique leaf $M_{\bar{c}}=\overline{p x}$ and a Fatou gap $V_{\bar{c}}$ such that $V_{\bar{c}}$ is of period $k$ and $M_{\bar{c}}$ is its refixed leaf.

Now, construct a proper geolamination $\mathcal{L}^{p r}$ out of $\mathcal{L}_{\mathrm{QCP}}$ using Definition 1.48 and Lemma 1.49 . If QCP is a privileged qc-portrait for $\mathcal{L}^{p r}$, there is nothing to prove. Suppose that QCP is not a privileged qc-portrait for $\mathcal{L}^{p r}$. By definition the only way it can happen is when the set $Q$ is contained in a quadratic Fatou gap $U$ of $\mathcal{L}^{p r}$, and, moreover, the period of the periodic edge of $Q$ is greater than the period of the gap $U$. In this case we apply the map $\psi_{U}$ which sends $Q$ to a $\sigma_{2}$-critical leaf $\bar{c}$ with a periodic endpoint, say, $p$ of period $k>1$ ( $k>1$ exactly because QCP is not privileged for $\mathcal{L}_{\mathrm{QCP}}$ ). Then we use Proposition 3.6, find a $\sigma_{2}$-periodic Fatou gap $V$ for which $p$ is an endpoint of a refixed edge, and pull $V$ back to $U$ using the 
projection map $\psi_{U}$. This finally produces a proper geolamination for which QCP is a privileged qc-portrait.

Theorem 3.7. Every $\mathrm{QCP}=(Q, D) \in \mathcal{Q C P}_{3}^{n p}$ is a privileged qc-portrait of a geolamination from $\mathbb{L} \mathbb{P}_{3}^{n p}$.

Proof. We may assume that $Q$ has a periodic (possibly degenerate) edge $\ell_{Q}$ of vertex period $N$ and its sibling-edge $\hat{\ell}_{Q}$. Consider the family $\mathcal{A}_{\mathrm{QCP}}$ of all cubic geolaminations containing $Q$ and $D$ (by Theorem $1.19 \mathcal{A}_{\mathrm{QCP}} \neq$ $\emptyset$ ). Among geolaminations from $\mathcal{A}_{\mathrm{QCP}}$, choose a geolamination $\mathcal{L}$ with a maximal family of non-degenerate periodic leaves of periods at most $N$. Clearly, such a geolamination $\mathcal{L}$ exists. By Lemma 1.49 we can find a proper geolamination $\mathcal{L}^{p r} \subset \mathcal{L}$ with $\ell_{Q} \in \mathcal{L}^{p r}$.

By way of contradiction we may assume that QCP is not privileged for $\mathcal{L}^{p r}$. Then there must exist a periodic Fatou gap $U_{Q}$ which contains $Q$. On the other hand, by Lemma $1.49 D$ is a leaf of $\mathcal{L}^{p r}$. It follows that $U_{Q}$ is a Fatou gap such that $\left.\sigma_{3}\right|_{\operatorname{Bd}\left(U_{Q}\right)}$ is of degree 2. Consider the case when $\ell_{Q}$ is non-degenerate. Since by Lemma $1.49 \ell_{Q} \in \mathcal{L}^{p r}$ then $\ell_{Q}$ is an edge of $U_{Q}$. Let us show that $\hat{\ell}_{Q} \in \mathcal{L}^{p r}$. Indeed, by Lemma 1.49 we include in $\mathcal{L}^{p r}$ those leaves of $\mathcal{L}$ which have disjoint sibling collections consisting of proper leaves and whose all images have the same property. Now, take $\hat{\ell}_{Q}$ and its disjoint sibling collection (which must exist by definition of a sibling invariant geolamination). It follows that such collection must include $\ell_{Q}$. Hence $\hat{\ell}_{Q} \in \mathcal{L}^{p r}$ as desired. By construction, $\ell_{Q}$ and $\hat{\ell}_{Q}$ are edges of $U_{Q}$. Now, if $\ell_{Q}$ is degenerate then $Q$ can be a critical leaf or an all-critical triangle (viewed as a generalized quadrilateral). In either case $\ell_{Q}$ is a vertex of (an edge of) $U_{Q}$ and $\hat{\ell}_{Q}$ is either a vertex of $U_{Q}$ or an edge of $U_{Q}$ (the latter holds if $Q$ is an all-critical triangle).

Let us show that then $\mathcal{L}^{p r}$ cannot be a Siegel geolamination of capture type. Indeed, suppose otherwise. Then $U_{Q}$ eventually maps onto a periodic Siegel gap, and so the original set $Q$ has edges with non-preperiodic leaves, a contradiction. Hence $\mathcal{L}^{p r}$ is not a Siegel geolamination of capture type.

The map $\phi_{U_{Q}}: \operatorname{Bd}\left(U_{Q}\right) \rightarrow \mathbb{S}$ collapses concatenations of edges in $\operatorname{Bd}\left(U_{Q}\right)$ to points and semiconjugates the remap of $\operatorname{Bd}\left(U_{Q}\right)$ to $\sigma_{2}$. Clearly, $\phi_{U_{Q}}(Q)=\overline{a b}$ is a $\sigma_{2}$-critical leaf with a periodic endpoint, say, $z$. If $z=0$ then $\ell_{Q}$ is a refixed edge of $U_{Q}$ and we are done. Assume that $z \neq 0$ and bring this to a contradiction. Set $M=M_{\overline{a b}}$ as in Proposition 3.6, and use $\phi_{U_{Q}}^{-1}$ to pull the Fatou gap $V=V_{\overline{a b}}$ from Proposition 3.6 back to a gap $V_{Q} \subset U_{Q}$ by taking full $\psi_{U_{Q}}$-preimages of points from $V \cap \mathbb{S}$ and then taking the convex hull of such preimages. Then $\ell_{Q}$ is an edge of $V_{Q}$ and a periodic edge $M_{Q}$ of $V_{Q}$ of vertex period $N$ maps to $M$ under $\psi_{U}$. By construction, $Q \subset V_{Q}$ while $M_{Q}$ is a periodic leaf whose orbit consists of 
leaves which do not cross $Q$. This contradicts the choice of the geolamination $\mathcal{L}$ as a geolamination with the maximal number of non-degenerate periodic leaves of period at most $N$.

Proof of Theorem A. By Lemma 3.2 there is at least one and at most finitely many qc-portraits privileged for a given geolamination from $\mathbb{L P}_{3}^{n p}$. On the other hand, by Theorem 3.7 every qc-portrait $\mathrm{QCP} \in \mathcal{Q} \mathcal{C} \mathcal{P}_{3}^{n p}$ is a privileged qc-portrait of a geolamination from $\mathbb{L}_{3} \mathbb{P}_{3}^{n p}$.

Recall that for $D \in \mathcal{N} \mathcal{P}, \mathcal{S}_{D}$ is the collection of admissible qc-portraits with $D$ as the second element. To show that $\mathcal{S}_{D}$ is compact, let $\left(Q_{n}, D\right) \rightarrow$ $(Q, D)$. By definition the fact that $Q_{n}$ 's are generalized critical quadrilaterals implies that $Q$ is a generalized critical quadrilateral. Moreover, if $(Q, D)$ is not admissible then either there exist numbers $i, j$ with $\sigma_{3}^{i}(Q)$ crossing $\sigma_{3}^{j}(Q)$, or there exists a number $k$ such that $\sigma_{3}^{k}(Q)$ crosses $D$. In either case, the same crossing will have to take place for the corresponding images of $Q_{n}$ 's and $D$ contradicting the fact that $\left(Q_{n}, D\right)$ is admissible. By Proposition $3.4(Q, D)$ is admissible.

Notice that if QCP is a privileged qc-portrait of a proper geolamination $\mathcal{L}$ and all vertices of sets from QCP are non-periodic then QCP remains a privileged qc-portrait of the $\mathbb{L} \mathcal{Q}$-geolamination $\mathcal{L}_{\sim_{\mathcal{L}}}$ generated by $\mathcal{L}$.

\section{INTERSECTING MiNORS OF $\mathcal{Q C} \mathcal{P}_{3}^{n p}$}

By definition a proper family of chords $\mathcal{F}$ generates a lamination $\sim_{\mathcal{F}}$ and, if $\mathcal{F}=\mathcal{L}$ is also a $\left(\sigma_{d^{-}}\right)$invariant geolamination, then $\sim_{\mathcal{L}}$ is a $\left(\sigma_{d^{-}}\right)$invariant lamination. Throughout the rest of this section the following holds.

Standing Assumption. We fix a critical leaf $D=\overline{a b} \in \mathcal{N} \mathcal{P}$ with a positive arc $(a, b) \subset \mathbb{S}$ of length $\frac{1}{3}$, set $\sigma_{3}(D)=d$, and denote by $\Delta$ the allcritical triangle $\mathrm{CH}(a, b, v)$. Fix admissible qc-portraits $\left(Q_{1}, D\right)=\mathrm{QCP}_{1}$ and $\left(Q_{2}, D\right)=\mathrm{QCP}_{2}$ such that their minors $\sigma_{3}\left(Q_{1}\right)$ and $\sigma_{3}\left(Q_{2}\right)$ are nondisjoint. Assume that $\mathrm{QCP}_{i}$ is a privileged qc-portrait for a proper geolamination $\mathcal{L}_{i}$ for $i=1,2$ such that neither $\mathcal{L}_{1}$ nor $\mathcal{L}_{2}$ is of Siegel capture type. Set $\sim_{\mathcal{L}_{1}}=\sim_{1}, \sim_{\mathcal{L}_{2}}=\sim_{2}$. Let $\widehat{\mathcal{L}}_{1}=\mathcal{L}_{\sim_{1}}$ and $\widehat{\mathcal{L}}_{2}=\mathcal{L}_{\sim_{2}}$.

By definition, $Q_{1} \neq D, Q_{2} \neq D$. On the other hand, it is possible that $Q_{1}=\Delta$ or $Q_{2}=\Delta$.

Our aim is to prove Theorem B, i.e. to prove that $\sim_{1}=\sim_{2}$. If $Q_{1}$ and $Q_{2}$ are strongly linked or share a spike, tools from [BOPT14] apply and eventually imply the desired. However we need to prove Theorem B under weaker assumptions that $\sigma_{3}\left(Q_{1}\right)$ and $\sigma_{3}\left(Q_{2}\right)$ are non-disjoint. Thus we need to study the case when $\sigma_{3}\left(Q_{1}\right)$ and $\sigma_{3}\left(Q_{2}\right)$ are non-disjoint but $Q_{1}$ and $Q_{2}$ are neither strongly linked nor share a spike. Lemma 4.1 shows that this is an exceptional case. 
Lemma 4.1. Qc-portraits $\mathrm{QCP}_{1}$ and $\mathrm{QCP}_{2}$ are linked unless one of the sets $Q_{1}, Q_{2}$ coincides with $\left(a, x, v, x^{\prime}\right)$ while the other one coincides with $\left(b, y, v, y^{\prime}\right)$.

Proof. Let us show that if $\sigma_{3}\left(Q_{1}\right) \cap \sigma_{3}\left(Q_{2}\right) \neq\{d\}$ then $\mathrm{QCP}_{1}$ and $\mathrm{QCP}_{2}$ are linked. Observe that $\left.\sigma_{3}\right|_{[b, a]}$ is two-to-one except that $d$ has three preimages $a, b, v \in[b, a]$. Consider cases. Assume first that $\sigma_{3}\left(Q_{1}\right) \cap \sigma_{3}\left(Q_{2}\right) \cap \mathbb{D} \neq$ 0 . Since both minors have points inside $\mathbb{D}$, they are non-degenerate, and the generalized quadrilaterals $Q_{1}, Q_{2}$ are true quadrilaterals. If the minors do not coincide, it immediately follows from properties of $\left.\sigma_{3}\right|_{[b, a]}$ and the assumptions that $Q_{1}$ and $Q_{2}$ are strongly linked. If minors coincide and do not have $d$ as an endpoint, it follows that $Q_{1}=Q_{2}$ (and hence by definition $Q_{1}$ and $Q_{2}$ are strongly linked). Assume that $\sigma_{3}\left(Q_{1}\right)=\sigma_{3}\left(Q_{2}\right)=\overline{d x}$ and denote by $x^{\prime}, x^{\prime \prime} \in(b, a)$ two points with $\sigma_{3}\left(x^{\prime}\right)=\sigma_{3}\left(x^{\prime \prime}\right)=x$. Then either $Q_{1}=Q_{2}$, or otherwise we may assume that $Q_{1}=\left(a, x^{\prime}, v, x^{\prime \prime}\right)$ and $Q_{2}=\mathrm{CH}\left(b, x^{\prime}, v, x^{\prime \prime}\right)$. Clearly, in this case again $Q_{1}$ and $Q_{2}$ are strongly linked. Assume now that $\sigma_{3}\left(Q_{1}\right) \cap \sigma_{3}\left(Q_{2}\right)=\{y\}$ where $y \neq d$. Let $y^{\prime}, y^{\prime \prime} \in(b, a)$ so that $\left.\sigma_{3}\left(y^{\prime}\right)=\sigma_{3}{ }^{\prime \prime}\right)=y$. Then both $Q_{1}$ and $Q_{2}$ share the spike $\overline{y^{\prime} y^{\prime \prime}}$. By definition, in all these cases $\mathrm{QCP}_{1}$ and $\mathrm{QCP}_{2}$ are linked. If now $\sigma_{3}\left(Q_{1}\right) \cap \sigma_{3}\left(Q_{2}\right)=\{d\}$ then both $Q_{1}$ and $Q_{2}$ have either $\overline{a v}$ or $\overline{b v}$ as a spike. If either $Q_{1}$ or $Q_{2}$ is $\Delta, \mathcal{L}_{1}$ and $\mathcal{L}_{2}$ are linked. Hence one of the sets $Q_{1}, Q_{2}$ coincides with $\left(a, x, v, x^{\prime}\right)$ while the other one coincides with $\left(b, y, v, y^{\prime}\right)$.

For brevity, if $\mathrm{QCP}_{1}$ and $\mathrm{QCP}_{2}$ are not linked we will say that Case $V$ holds; without loss of generality we will then always assume that $Q_{1}=$ $\left(a, x, v, x^{\prime}\right)$ and $Q_{2}=\left(b, y, v, y^{\prime}\right)$. We use Lemma 4.1 to handle Case V. It turns out that then either Theorem B follows from known results, or specific restructuring allows us to find geolaminations which are linked and generate the same laminations as the given geolaminations. This show that it is enough to prove Theorem $\mathrm{B}$ in the case when $\mathrm{QCP}_{1}$ and $\mathrm{QCP}_{2}$ are linked.

Lemma 4.2. Suppose that Case Vholds and $v$ is not periodic. Then $\sim_{1}=\sim_{2}$.

Proof. Since $a, b$ and $v$ are not periodic, $Q_{1}$ and $Q_{2}$ cannot be critical quadrilaterals generated by periodic Fatou gaps. Hence $\sim_{1}\left(\sim_{2}\right)$ has a unique finite critical class $\mathfrak{g}_{1}\left(\mathfrak{g}_{2}\right)$ on which $\sigma_{3}$ is three-to-one. Clearly, $\{a, b, v\}$ is contained in both $\mathfrak{g}_{1}$ and $\mathfrak{g}_{2}$ so that $d=\sigma_{3}(a) \in \sigma_{3}\left(\mathfrak{g}_{1}\right) \cap \sigma_{3}\left(\mathfrak{g}_{2}\right)$. By [Sch04], $\mathfrak{g}_{1}=\mathfrak{g}_{2}$. A standard pull-back argument then shows that $\sim_{1}=\sim_{2}$.

To complete considering Case $\mathrm{V}$ we now assume that $v$ is periodic. We need a few lemmas from [CHMMO14] where they are proven in the degree $d$ case (we restate them for the cubic case). 
Lemma 4.3 (Lemma 1.5 [CHMMO14]). Suppose that $\mathcal{L}$ is a cubic geolamination. Then for any leaf $\ell=\overline{a b}$ of $\mathcal{L}$ there exists $i \geq 0$ such that both circle arcs with endpoints $\sigma_{3}^{i}(a), \sigma_{3}^{i}(b)$ are of length at least $\frac{1}{4}$.

Given a circle chord $\ell=\overline{x y}$ which is not a diameter, let $\|\ell\|$ be the length of the smallest of the two arcs in $\mathbb{S}$ with endpoints $x, y$. Let $\ell_{0}=\overline{a b}$ be a chord with $0<\left\|\ell_{0}\right\|=b-a<\frac{1}{3}$. Let $I_{0}=\left[b, a+\frac{1}{3}\right]$; let $I_{1}$ be $I_{0}$ rotated by $\frac{1}{3}$ and $I_{2}$ be $I_{0}$ rotated by $\frac{2}{3}$. Define the central strip $\operatorname{CS}\left(\ell_{0}\right)$ of $\ell_{0}$ as the convex hull of $I_{0} \cup I_{1} \cup I_{3}$.

Lemma 4.4 (Theorem 2.10 [CHMMO14]). Suppose that $\mathcal{L}$ is a cubic geolamination, $\ell$ is a leaf of $\mathcal{L}$ such that $\frac{1}{4} \leq\|\ell\|<\frac{1}{3}$. Let $j>0$ be the least number such that $\sigma_{3}^{j}(\ell)$ has an endpoint in $\operatorname{CS}(\ell)$. Then $\sigma_{3}^{j}(\ell)$ has endpoints in two distinct circle arcs on the boundary of $\mathrm{CS}(\ell)$.

We use these tools to prove the next lemma.

Lemma 4.5. Let a proper geolamination $\mathcal{L}$ have a periodic critical Fatou gap $U$ of degree two and a refixed edge $\ell$. Let $\ell^{\prime}$ be the sibling of $\ell$ contained in $\operatorname{Bd}(U)$. If $\ell^{\prime}$ is contained in a finite critical gap $G^{\prime}$ of $\sim_{\mathcal{L}}$ then $\ell$ is the unique refixed edge of $U$ and $\frac{1}{4} \leq\|\ell\|<\frac{1}{3}$. Moreover, the entire orbit of $U$ except for $U$ itself is disjoint from $\mathrm{CS}(\ell)$.

A weaker version of the last claim of the lemma, which may be easier to apply, is that the images of $\ell$ do not intersect the two all-critical triangles one of whose vertices is an endpoint of $\ell$.

Proof. Suppose that $\ell=\overline{p q}$. Then $p+\frac{1}{3}, p+\frac{2}{3} \in G^{\prime}$. Since $\ell$ does not cross edges or diagonals of $G^{\prime},\|\ell\| \leq \frac{1}{3}$. Moreover, since $\ell$ is refixed, $\|\ell\|<\frac{1}{3}$. Let $T$ be the all-critical triangle with vertex $p$. Clearly, images of $\ell$ or any other periodic edge of $U$ do not cross edges of $T$. Consider now any refixed edge $\hat{\ell}$ of $U$ and prove that $\|\hat{\ell}\| \geq \frac{1}{4}$.

Indeed, if $\|\hat{\ell}\|<\frac{1}{4}$, choose $i$ with $\left\|\sigma_{3}^{i}(\hat{\ell})\right\|$ maximal. Clearly, $T \subset$ $\operatorname{CS}\left(\sigma_{3}^{i}(\hat{\ell})\right)$. By Lemma 4.3 we have that $\frac{1}{4} \leq\left\|\sigma_{3}^{i}(\hat{\ell})\right\|<\frac{1}{3}$, the set $\operatorname{CS}\left(\sigma_{3}^{i}(\hat{\ell})\right)$ is well-defined, and $\sigma_{3}^{i}(\hat{\ell}) \neq \hat{\ell}$. Since $\hat{\ell}$ is itself an eventual image of $\sigma_{3}^{i}(\hat{\ell})$, $\sigma_{3}^{i}(\hat{\ell})$ at some later moment enters $\operatorname{CS}\left(\sigma_{3}^{i}(\hat{\ell})\right)$. However, by Lemma 4.4 the first time $\sigma_{3}^{i}(\hat{\ell})$ enters $\operatorname{CS}\left(\sigma_{3}^{i}(\hat{\ell})\right)$, the corresponding image of $\sigma_{3}^{i}(\hat{\ell})$ will have its endpoints in distinct arcs of the boundary of $\operatorname{CS}\left(\sigma_{3}^{i}(\hat{\ell})\right)$, a contradiction with maximality of $\left\|\sigma_{3}^{i}(\hat{\ell})\right\|$. Hence $\|\hat{\ell}\| \geq \frac{1}{4}$, and the circle arcs on the boundary of $\operatorname{CS}(\hat{\ell})$ are of length at most $\frac{1}{3}-\frac{1}{4}=\frac{1}{12}$. Hence $\ell$ is the unique refixed edge of $U$. The last claim of the lemma follows from Lemma 4.4 . 
Now we can go back to the case of two proper geolaminations for which Case V holds.

Lemma 4.6. Suppose that Case V holds and $v$ is periodic. Then we can replace the quadrilateral $Q_{2}$ by a quadrilateral $Q_{2}^{\prime}$ so that the new qc-portrait $\left(Q_{2}^{\prime}, D\right)$ is still privileged for $\mathcal{L}_{2}$ and is linked with $\mathrm{QCP}_{1}$.

Proof. Since $v$ is periodic and $\mathrm{QCP}_{2}$ is privileged for $\mathcal{L}_{2}$, either $\overline{y v}$ or $\overline{v y^{\prime}}$ is a refixed edge of a periodic Fatou gap $U$ of degree two. Assume that $\overline{v y^{\prime}}$ is a refixed edge of $U$. Then the leaves $\overline{v y}, \overline{b y^{\prime}}$ are not leaves of $\mathcal{L}_{2}$.

By Lemma $4.5 \overline{v y^{\prime}}$ is actually the unique refixed edge of $U$. Let us show that then no edge of $U$ crosses the critical chord $\overline{v a}$. Indeed, suppose otherwise. Clearly, the only way an edge $\ell$ of $U$ can cross $\overline{v a}$ is when it has $b$ as an endpoint. By [BMOV13] this implies that $\mathcal{L}$ has a leaf $\overline{z v}$ with $b<z<v$ which is a sibling leaf of $\ell$. Since $\mathcal{L}_{2}$ is proper, the leaf $\overline{v z}$ must be periodic of the same period as $\ell$. Since $\ell$ is the unique refixed edge of $U$, the leaf $\overline{z v}$ cannot be an edge of $U$. This implies that for geometric reasons there still must exist an edge of $U$ coming out of $v$ and different from $\overline{v y}$. However, as above, this edge of $U$ will then have to be refixed, again contradicting Lemma 4.5 .

Thus, we can remove $\overline{v y}$ and $\overline{y^{\prime} b}$ (i.e., two sides of $Q_{2}$ which are not edges of $U$ ) and replace them by the critical leaf $\overline{v a}$. By definition, the qc-portrait $(\overline{v a}, D)$ is privileged for $\mathcal{L}_{2}$. On the other hand, by definition $(\overline{v a}, D)$ is linked with $\mathrm{QCP}_{1}$. This completes the proof.

Thus, to prove Theorem B it suffices to consider the case when $Q_{1}, Q_{2}$ are linked as by Lemma 4.6 Case V can be reduced to this case. So, from now on we may assume that $Q_{1}, Q_{2}$ are linked and therefore $\left(\mathcal{L}_{1}, Q_{1}\right)$ and $\left(\mathcal{L}_{2}, Q_{2}\right)$ are linked.

We will need Lemma 4.8 from [BMOV13].

Lemma 4.7 (Lemma 4.8 [BMOV13]). If $\mathcal{L}$ is a $\sigma_{d}$-invariant geolamination and $\ell \in \mathcal{L}$ is a leaf such that $\sigma_{d}^{n}(\ell)$ is concatenated with $\ell$ for some $n$ then the endpoints of $\ell$ are (pre)periodic.

Proposition 4.8 studies possible intersections between various leaves and edges of $\Delta$.

Proposition 4.8. If $\mathrm{QCP}=(Q, D) \in \mathcal{S}_{D}$ with $d \in \sigma_{3}(Q)$, then no eventual $\sigma_{3}$-image of $Q$ crosses an edge of $\Delta$. If, moreover, $\mathcal{L}$ is a proper geolamination for which $\mathrm{QCP}$ is privileged, then no periodic leaf of $\mathcal{L}$ crosses an edge of $\Delta$.

Proof. Let $\overline{a v}$ be a spike of $Q$. To prove the first claim of the lemma, assume that $Q=\left(a, x, v, x^{\prime}\right)$ is a true quadrilateral, and show that no eventual $\sigma_{3^{-}}$ image of $Q$ crosses $\overline{b v}$. Indeed, suppose that $\sigma_{3}^{k}(Q)$ crosses $\overline{b v}$. Then, since 
images of $Q$ cannot cross $D$ or $Q$, it follows that the leaf $\sigma_{3}^{k}(Q)$ equals $\overline{a y}$ and is concatenated with the leaf $\overline{x a}$. By Lemma 4.7, points $a$ and $y$ are (pre)periodic. By the assumptions $a$ is not periodic. By Corollary 3.5, $\overline{a y}$ and all its images are proper leaves. However if we now follow the concatenation of leaves $\overline{a y}, \sigma_{3}^{k}(\overline{a y})$ etc, we will at some point encounter the situation when exactly one endpoint of some image of $\overline{a y}$ is periodic, a contradiction.

Consider the above situation for the sake of definiteness. Assume that QCP is a privileged qc-portrait of a cubic proper geolamination $\mathcal{L}$; then leaves of $\mathcal{L}$ cannot cross $\overline{a v}$ or $D$. Hence if a leaf $\ell$ of $\mathcal{L}$ crosses an edge of $\Delta$ then $\ell=\overline{a z}$ which cannot be periodic because $a$ is not periodic.

For a point $z \in \mathbb{S}$, let $F(z)$ be the set of endpoints of leaves from both $\mathcal{L}_{1}$ and $\mathcal{L}_{2}$ containing $z$. Note that $z \in F(z)$. Recall that by Lemma 1.40 (3) if an endpoint of a leaf $\ell$ of a proper geolamination is periodic, the other endpoint of $\ell$ is also periodic of the same period. Thus, the notion of vertex period is well-defined for periodic leaves.

Lemma 4.9. If $z \in \mathbb{S}$ is periodic under $\sigma_{3}$, then $\left.\sigma_{3}\right|_{F(z)}$ is order preserving.

Proof. By Corollary 1.16 and since $\mathcal{L}_{1}, \mathcal{L}_{2}$ are proper, all points in $F(z)$ are periodic of the same period. It suffices to show that for any distinct points $x, y \neq z$ of $F(z)$ the points $x, y, z$ are kept in order by $\sigma_{3}$. By Lemma 1.11 we may assume that $\overline{x z} \in \mathcal{L}_{1}$ and $\overline{y z} \in \mathcal{L}_{2}$. We show that there is only one point such that when $z$ coincides with this point it becomes possible for the order among the points $x, y, z$ to be reversed under $\sigma_{3}$.

First let $Q_{1}, Q_{2}$ be neither strongly linked nor share a spike. By Lemma 4.1 we may assume that $Q_{1}$ has a spike $\overline{a v}$ and $Q_{2}$ has a spike $\overline{b v}$. If $z \neq v$ then $z \notin \Delta$. By Proposition $4.8, \overline{x z}, \overline{y z}$ do not cross $\Delta$. Hence $x, y, z$ belong either to $[a, b]$, or to $[b, v]$, or to $[v, a]$. Also, $a, b \notin\{x, y, z\}$ because $a, b$ are non-periodic. It follows that $\left.\sigma_{3}\right|_{\{x, y, z\}}$ preserves the order. Hence in this case the unique location for $z$ for which the order among $a, y, z$ can be reversed is when $z=v$.

It remains to consider the case when $Q_{1}$ and $Q_{2}$ are strongly linked or share a spike. In this case the qc-portraits $\mathrm{QCP}_{1}, \mathrm{QCP}_{2}$ are linked and by Lemma 1.31 the order among the points $x, y, z$ can be reversed only if $z$ is a common vertex of associated quadrilaterals. If there is a unique such periodic vertex, then the claim is proven. Assume now that $Q_{1}$ and $Q_{2}$ have more than one common periodic vertex. If so, then both $Q_{1}$ and $Q_{2}$ must have at least three vertices (because for any critical chord only one of its endpoints can be periodic). If, say, $Q_{1}$ is a triangle then it must coincide with $\Delta$, hence in that case the unique location of $z$ which allows for the reversal of orientation is $z=v$, and the claim is once again verified. 
Consider finally the case when both $Q_{1}$ and $Q_{2}$ are quadrilaterals sharing an edge $\overline{u t}$ with both endpoints periodic. Let $\mathfrak{g}_{i}$ be the class of $\sim_{i}$ which contains $u$ and $t$. Since the class $\mathfrak{g}_{i}$ is periodic and finite, it is disjoint from $\overline{a b}$. Hence the siblings of $u$ and $t$ in $(b, a)$ are unique and $Q_{1}=Q_{2}=Q$ is the same quadrilateral. Let us show that then $\left.\sigma_{3}\right|_{\{x, y, z\}}$ is order preserving. We may assume that $z=u$. Then there are two cases. First, the points $x$ and $y$ may be separated by the spike of $Q$ with an endpoint $u$. Then the remaining spike of $Q$ and $D$ cut $\bar{D}$ in pieces one of which contains $x, y$ and $z$ which implies that $\left.\sigma_{3}\right|_{\{x, y, z\}}$ is order preserving. Otherwise the points $x$ and $y$ are not separated by the spike of $Q$ with an endpoint $u$. Then this very spike and $D$ again cut $\overline{\mathbb{D}}$ in pieces one of which contains $x, y$ and $z$ which implies that $\left.\sigma_{3}\right|_{\{x, y, z\}}$ is order preserving. By induction $\left.\sigma_{3}^{k}\right|_{\{x, y, z\}}$ is order preserving for all $k$.

Thus, there is a unique location of $z$ for which the orientation of the points $x, y, z$ can be reversed. However the periods of point $x, y, z$ are the same, hence the power of $\sigma_{3}$ which maps $z$ back to itself sends both $x$ and $y$ back to themselves too. Since by the above claim the reversal of orientation can happen along the way only once, it follows that it does not take place at all. We conclude that the claim holds for all periodic $z$.

Suppose that say, $\mathrm{CH}\left(Q_{1}\right)=\Delta$. By definition either $Q_{1}=(a, b, b, v)$, or $Q_{1}=(a, b, v, v)$, or $Q_{1}=(a, a, b, v)$. Then either $\Delta$ is a gap of $\mathcal{L}_{2}$ as well, or $Q_{1}$ and $Q_{2}$ are strongly linked, or $Q_{1}$ and $Q_{2}$ share a spike. Let us show that either $\overline{v a}$ or $\overline{b v}$ is a spike of $Q_{2}$. This is obvious if $\Delta$ is a gap of $\mathcal{L}_{2}$. Suppose that $\Delta$ is not a gap of $\mathcal{L}_{2}$ and neither $\overline{v a}$ nor $\overline{b v}$ is a spike of $Q_{2}$. Since by definition $\mathrm{CH}\left(Q_{2}\right) \neq D$, then it is easy to see that no vertex of $Q_{2}$ may coincide with $a, b$ or $v$. However then it follows that $Q_{2}$ and $Q_{1}$ cannot be strongly linked. Thus, if $\mathrm{CH}\left(Q_{1}\right)=\Delta$ then we can always assume that either $\overline{v a}$ or $\overline{b v}$ is a spike of $Q_{2}$.

Proposition 4.10 studies vertex periods of linked leaves of $\mathcal{L}_{1}, \mathcal{L}_{2}$.

Proposition 4.10. Suppose that a leaf $\ell_{1} \in \mathcal{L}_{1}$ is periodic of vertex period $n$ and a leaf $\ell_{2} \in \mathcal{L}_{2}$ meets $\ell_{1}$. Then the order among the endpoints of $\ell_{1}$ and $\ell_{2}$ is preserved under $\sigma_{3}$ and $\ell_{2}$ is periodic with vertex period $n$.

Proof. If $\ell_{1}$ and $\ell_{2}$ meet at an endpoint, the claim follows from Lemma 4.9. Otherwise we may assume that $\ell_{1}=\overline{p_{1} q_{1}}, \ell_{2}=\overline{p_{2} q_{2}}$ and $p_{1}<p_{2}<q_{1}<q_{2}$. Observe that $\ell_{2}$ cannot be critical. Indeed, suppose otherwise. Since $\ell_{2}$ and $\ell_{1}$ cross, $\ell_{2} \neq D$. Then there are the following options for $Q_{2}: \operatorname{CH}\left(Q_{2}\right)=$ $\Delta, \mathrm{CH}\left(Q_{2}\right)$ is the edge of $\Delta$ not equal to $D$ or $\ell_{2}$, and finally $\mathrm{CH}\left(Q_{2}\right)=\ell_{2}$. Let us first assume that $\mathrm{CH}\left(Q_{2}\right)=\ell_{2}=\overline{s t}$. Then $Q_{2}=(s, s, t, t)$. Since $\ell_{1}$ crosses $\ell_{2}, Q_{1}$ must be located on one side of $\ell_{1}$. However, this would 
imply that $Q_{1}$ and $Q_{2}$ can neither be strongly linked nor share a spike, a contradiction.

Thus, either $\mathrm{CH}\left(Q_{2}\right)=\Delta$, or $\mathrm{CH}\left(Q_{2}\right)$ is the edge of $\Delta$ not equal to $D$ or $\ell_{2}$, and $\ell_{2}$ is either $\overline{b v}$ or $\overline{v a}$. Observe that in either case either $a, a$, or $a, b$, or $b, b$ are consecutive vertices of $Q_{2}$. On the other hand, the leaf $\ell_{1}$ crosses $\ell_{2}$ and, therefore, is linked with both $\overline{b v}$ and $\overline{v a}$. As before, it means that $Q_{1}$ is located on one side of $\ell_{1}$ and hence, by definition, $Q_{1}$ cannot be linked with $Q_{2}$, a contradiction. So we may assume that $\ell_{2}$ is not critical.

Let us show that the order among the endpoints of $\ell_{1}, \ell_{2}$ is preserved. Indeed, suppose otherwise. By Lemma 1.31, without loss of generality we may assume that $\sigma_{3}\left(p_{1}\right)=\sigma_{3}\left(q_{2}\right)$. By Lemma 1.33 there is a sibling $\overline{p_{1} p_{2}^{\prime}}$ of $\overline{p_{2} q_{2}}$ with $q_{2}<p_{2}^{\prime}<p_{1}$. Since $\mathcal{L}_{2}$ is proper, $p_{2}^{\prime}$ is periodic. Then by Lemma 4.9. $\sigma_{3}\left(p_{2}^{\prime}\right)=\sigma_{3}\left(p_{2}\right)<\sigma_{3}\left(p_{1}\right)<\sigma_{3}\left(q_{1}\right)$. On the other hand, since $\left(\mathcal{L}_{1}, \mathrm{QCP}_{1}\right),\left(\mathcal{L}_{2}, \mathrm{QCP}_{2}\right)$ are linked, then by Lemma 1.31 the order among the points $p_{1}, p_{2}, q_{1}, q_{2}$ is weakly preserved under $\sigma_{3}$ so that $\sigma_{3}\left(p_{1}\right) \leq$ $\sigma_{3}\left(p_{2}\right) \leq \sigma_{3}\left(q_{1}\right)$, a contradiction. Thus, the order among the endpoints of $\ell_{1}, \ell_{2}$ is preserved. In particular, $\ell_{2}$ is not (pre)critical.

Let us show that $\ell_{2}$ is (pre)periodic. Consider $A_{\ell_{2}}\left(\ell_{1}\right)=A$. By the above, the order of endpoints of $\ell_{1}, \ell_{2}$ is preserved under $\sigma_{3}^{n k}$ for every $k \geq 0$. Hence the sequence of points of intersection of leaves $\sigma_{3}^{n k}\left(\ell_{2}\right)$ with $\ell_{1}$ is monotone on $\ell_{1}$ while all leaves $\sigma_{3}^{n k}\left(\ell_{2}\right)$ are pairwise unlinked. Then there exists a leaf $\ell_{\infty}$ equal to the limit of leaves $\sigma_{3}^{n k}\left(\ell_{2}\right)$. Since $\sigma_{3}$ is locally expanding, $\ell_{2}$ is (pre)periodic. Moreover, by Lemma 1.26 the vertex period of the eventual periodic image of $\ell_{2}$ is $n$.

Let us now show that in fact $\ell_{2}$ is periodic itself. Suppose otherwise. Then for some $i, \sigma_{3}^{i}\left(\ell_{2}\right)$ and $\sigma_{3}^{n+i}\left(\ell_{2}\right)$ are disjoint siblings which are both linked with $\sigma_{3}^{i}\left(\ell_{1}\right)$. Clearly, $D$ does not separate $\sigma_{3}^{i}\left(\ell_{2}\right)$ and $\sigma_{3}^{n+i}\left(\ell_{2}\right)$ as otherwise $D$ and $\ell_{1}$ would cross. Thus, $\sigma_{3}^{i}\left(\ell_{2}\right)$ and $\sigma_{3}^{n+i}\left(\ell_{2}\right)$ are located in the closure $\bar{W}$ of the component $W$ of $\overline{\mathbb{D}} \backslash D$ with boundary circle arc of length $\frac{2}{3}$. Moreover, since $\sigma_{3}^{i}\left(\ell_{2}\right)$ and $\sigma_{3}^{n+i}\left(\ell_{2}\right)$ are disjoint siblings, both spikes of $Q_{2}$ must separate $\sigma_{3}^{i}\left(\ell_{2}\right)$ and $\sigma_{3}^{n+i}\left(\ell_{2}\right)$. Thus, both spikes of $Q_{2}$ cross $\ell_{1}$. Since $Q_{1}$ and $Q_{2}$ are strongly linked or share a spike, it follows that $Q_{1}$ has a spike linked with $\ell_{1}$, a contradiction. Thus, $\ell_{2}$ is periodic.

Observe that if $\mathcal{L}$ is proper then all $\sim_{\mathcal{L}}$-classes are finite. Hence for any point $x \in \mathbb{S}$ there are at most finitely many leaves of $\mathcal{L}$ containing $x$.

Proposition 4.11. No leaf $\ell_{1} \in \mathcal{L}_{1}$ (resp. $\ell_{2} \in \mathcal{L}_{2}$ ) can intersect infinitely many leaves of $\mathcal{L}_{2}$ (resp. $\left.\mathcal{L}_{1}\right)$. In particular, no leaf $\ell_{1} \in \mathcal{L}_{1}$ (resp. $\left.\ell_{2} \in \mathcal{L}_{2}\right)$ is linked with a limit leaf of $\mathcal{L}_{2}$ (resp. $\left.\mathcal{L}_{1}\right)$.

Proof. If $\ell_{1} \in \mathcal{L}_{1}$ intersects infinitely many leaves $\left\{\ell_{2}^{j}\right\}_{1}^{\infty}$ of $\mathcal{L}_{2}$ then by Lemma $1.31 \sigma_{3}^{i}\left(\ell_{1}\right)$ intersects $\sigma_{3}^{i}$-images of all leaves $\left\{\ell_{2}^{j}\right\}_{1}^{\infty}$ for any $i$. Since 
for any point $x \in \mathbb{S}$ there are at most finitely many leaves of $\mathcal{L}$ containing $x, \sigma_{3}^{i}\left(\ell_{1}\right)$ is linked with infinitely many leaves of $\mathcal{L}_{2}$. If $\sigma_{3}^{i}\left(\ell_{1}\right)$ is periodic for some $i$ this would contradict Proposition 4.10. Thus we may assume that the orbits of the endpoints of $\ell_{1}$ are infinite. As there are only finitely many chains of spikes of $\mathcal{L}_{1}$ or $\mathcal{L}_{2}$, for some $i_{0}$ and any $i \geq i_{0}$ the leaf $\sigma_{3}^{i}\left(\ell_{1}\right)$ cannot collapse around any chain of spikes. Since $\sigma_{3}^{i_{0}}\left(\ell_{1}\right)$ is linked with infinitely many leaves of $\mathcal{L}_{2}$, Lemma 1.31 implies that $\sigma_{3}^{i_{0}}\left(\ell_{1}\right)$ and those leaves of $\mathcal{L}_{2}$ have mutually order preserving accordions. Since true quadrilaterals cannot be wandering under $\sigma_{3}$ [Kiw02], by Theorem $1.29 \ell_{1}$ is (pre)periodic, a contradiction.

Let us study Fatou gaps of our geolaminations. For a Fatou gap $U$ of $\mathcal{L}_{1}$, let $\phi_{U}: \partial U \rightarrow \mathbb{S}$ be the map collapsing all leaves in $\operatorname{Bd}(U)$ to points. For a leaf $\ell_{2} \in \mathcal{L}_{2}$ with $\ell_{2} \cap \operatorname{Bd}(U) \neq 0$, let $\ell^{\prime}, \ell^{\prime \prime}$ be edges of $\operatorname{Bd}(U)$ which intersect $\ell_{2}$ such that $\phi_{U}\left(\ell^{\prime}\right) \neq \phi_{U}\left(\ell^{\prime \prime}\right)$ if possible. If so, define $\phi_{U}\left(\ell_{2}\right)=$ $\overline{\phi_{U}\left(\ell^{\prime}\right) \phi_{U}\left(\ell^{\prime \prime}\right)}$ and define $\phi_{U}\left(\ell_{2}\right)=\phi_{U}\left(\ell_{2} \cap \operatorname{Bd}(U)\right)$ otherwise. Observe that the map $\phi_{U}$ is defined not only for periodic but also for non-periodic Fatou gaps (recall that all Fatou gaps are (pre)periodic).

Lemma 4.12. If $U$ is a Fatou gap of $\mathcal{L}_{1}$, then, for any leaf $\ell_{2} \in \mathcal{L}_{2}$ such that $\ell_{2} \cap \partial U \neq \emptyset$, the set $\phi_{U}\left(\ell_{2}\right)$ is degenerate.

Proof. Assume that $\phi_{U}\left(\ell_{2}\right)$ is non-degenerate. Let us show that we may assume that $U$ is periodic. Indeed, any Fatou gap eventually maps to a periodic Fatou gap. Moreover, since $\mathcal{L}_{1}$ is not of capture Siegel type then there are no critical preperiodic Fatou gaps (in our case, i.e. with $D$ being a part of $\mathcal{L}_{1}$, a critical preperiodic Fatou gap which maps to a periodic Fatou gap of degree greater than one is impossible). Thus, if $W$ is a non-periodic Fatou gap of $\mathcal{L}_{1}$ and $\bar{h}$ and $\bar{k}$ are its edges which do not belong to the same concatenation of edges of $W$ then their images are distinct. Hence, if $\phi_{U}\left(\ell_{2}\right)$ is not degenerate, we can keep mapping $U$ forward until $U$ maps to a periodic gap $W$ and consider intersections of images $\ell_{2}$ with appropriate images of $U$. By Lemma 1.31 the intersections of $\ell_{2}$ with edges of $U$ are preserved under iteration of $\sigma_{3}$. Since along the way to $W$ all preperiodic images of $U$ are non-critical, we conclude that the image of $\ell_{2}$ which intersects $W$ gives rise to a non-degenerate $\phi_{W}$-image. Thus, we can assume without loss of generality that $U$ is periodic from the very beginning.

Now, for geolaminations of $\mathbb{L} \mathbb{P}_{3}^{n p}$, all periodic Fatou gaps are of degree 1 or 2 . Assume that $U$ is of degree 2. Then $Q_{1}$ has an edge $M$ which is a refixed edge of $U$. Denote the sibling edge of $M$ in $U$ by $M^{\prime}$. Thus, $\phi_{U}\left(Q_{1}\right)=\overline{0 \frac{1}{2}}$. Since $\sigma_{3}$-images of $\ell_{2}$ do not cross one another, $\sigma_{2}$-images of $\phi_{U}\left(\ell_{2}\right)$ do not cross one another either. Then there exists $k$ such that either $(1) \sigma_{2}^{k}\left(\phi_{U}\left(\ell_{2}\right)\right)$ crosses $\overline{0 \frac{1}{2}}$, or (2) $\sigma_{2}^{k}\left(\phi_{U}\left(\ell_{2}\right)\right)=\overline{0 \frac{1}{2}}$. If (1) holds, then 
$\sigma_{3}^{k}\left(\ell_{2}\right)$ crosses two improper leaves of $Q_{1}$, contradicting the fact that $Q_{2}$ is strongly linked with $Q_{1}$ or shares a spike with $Q_{1}$. If (2) holds, $\sigma_{3}^{k}\left(\ell_{2}\right)$ meets one refixed edge of $U$ and one edge of $U$ which is not periodic contradicting Proposition 4.10 (indeed, if $\sigma_{3}^{k}\left(\ell_{2}\right)$ is not periodic it cannot meet any refixed edge of $U$ while if $\sigma_{3}^{k}\left(\ell_{2}\right)$ is periodic it cannot meet non-periodic edge of $U)$. Finally, if $U$ is of degree 1 then $\phi_{U}\left(\ell_{2}\right)$ will eventually cross itself under irrational rotation induced on $\phi_{U}(\operatorname{Bd}(U))=\mathbb{S}$, a contradiction.

Recall that $\widehat{\mathcal{L}}_{1}$ and $\widehat{\mathcal{L}}_{2}$ are canonical geolaminations constructed for laminations $\sim_{1}, \sim_{2}$ in turn generated by the given geolaminations $\mathcal{L}_{1}, \mathcal{L}_{2}$.

Proof of Theorem B. First we prove that Fatou gaps of $\widehat{\mathcal{L}}_{1}$ and $\widehat{\mathcal{L}}_{2}$ are the same. A Fatou gap of either geolamination $\widehat{\mathcal{L}}_{i}$ cannot have a non-trivial (consisting of more than one) concatenation of its edges as it will have to be completed with an edge separating it from the rest of the Fatou gap in question. Let $\widehat{U}_{1}$ be a periodic Fatou gap of $\widehat{\mathcal{L}}_{1}$. The corresponding Fatou gap $U_{1}$ of $\mathcal{L}_{1}$ is of the same degree as $\widehat{U}_{1}$. If for a leaf $\hat{\ell}_{2} \in \widehat{\mathcal{L}}_{2}$ the chord $\phi_{\widehat{U}_{1}}\left(\hat{\ell}_{2}\right)$ is non-degenerate, then there must exist a leaf $\ell_{2} \in \mathcal{L}_{2}$ with nondegenerate image $\phi_{U_{1}}\left(\ell_{2}\right)$, contradicting Lemma 4.12. Hence there exists an infinite gap $\widehat{U}_{2}$ of $\widehat{\mathcal{L}}_{2}$ containing $\widehat{U}_{1}$. Similarly, there exists an infinite gap of $\widehat{\mathcal{L}}_{1}$ containing $\widehat{U}_{2}$. Thus, $\widehat{U}_{1}=\widehat{U}_{2}$.

We claim now that leaves of $\widehat{\mathcal{L}}_{1}$ and $\widehat{\mathcal{L}}_{2}$ coincide. Call a leaf of $\widehat{\mathcal{L}}_{i}$ a limit leaf if it is the limit of leaves of $\widehat{\mathcal{L}}_{i}$. We claim the limit leaves of $\widehat{\mathcal{L}}_{1}$ and the limit leaves of $\widehat{\mathcal{L}}_{2}$ form the same family of leaves. Indeed, let $\hat{\ell}_{1} \in \widehat{\mathcal{L}}_{1}$ be a limit leaf, and prove that then $\hat{\ell}_{1}$ is a leaf of $\widehat{\mathcal{L}}_{2}$. Observe that $\hat{\ell}_{1}$ must be a leaf of $\mathcal{L}_{1}$ too. By Proposition $4.11 \hat{\ell}_{1}$ is not linked with any leaf of $\mathcal{L}_{2}$ as any such leaf of $\mathcal{L}_{2}$ will be crossed by infinitely many leaves of $\mathcal{L}_{1}$. This easily implies that $\hat{\ell}_{1}$ in fact is not linked with leaves of $\widehat{\mathcal{L}}_{2}$ either. Moreover, the same arguments show that no leaf of $\widehat{\mathcal{L}}_{2}$ can share an endpoint with $\hat{\ell}_{1}$ and be otherwise located on the side of $\hat{\ell}_{1}$ from which $\hat{\ell}_{1}$ is approached by infinitely many leaves of $\widehat{\mathcal{L}}_{1}$. Suppose that $\hat{\ell}_{1}$ is not a leaf of $\widehat{\mathcal{L}}_{2}$. Then by the above $\hat{\ell}_{1}$ is contained (except the endpoints) in a Fatou gap $V$ of $\widehat{\mathcal{L}}_{2}$, a contradiction with the above.

On the other hand, suppose that $\hat{\ell}_{1} \in \widehat{\mathcal{L}}_{1}$ is not a limit leaf of $\widehat{\mathcal{L}}_{1}$. Then on at least one side a Fatou gap is attached to $\hat{\ell}_{1}$ which implies that $\hat{\ell}_{1}$ is a leaf of $\widehat{\mathcal{L}}_{2}$ too. Thus, $\widehat{\mathcal{L}}_{1}=\widehat{\mathcal{L}}_{2}$ as desired.

\section{Unlinkage of QC-Portraits of $\mathcal{Q} \mathcal{C} \mathcal{P}_{3}^{n p}$}

In what follows we always assume that $\mathrm{QCP}_{1}=\left(Q_{1}, D\right), \mathrm{QCP}_{2}=$ $\left(Q_{2}, D\right) \in \mathcal{Q C P}_{3}^{n p}$ are linked and distinct privileged portraits of proper geolaminations $\mathcal{L}_{1}, \mathcal{L}_{2} \in \mathcal{S}_{D}$. Recall that $\Delta=\mathrm{CH}(a, b, v)$ is the all-critical 
triangle with edge $D=\overline{a b}$ where $(a, b)$ is a circle arc of length $\frac{1}{3}$. Observe that $(\Delta, D)$ is an admissible qc-portrait.

Lemma 5.1. If $Q_{1}=\Delta$, then $Q_{2}$ is an edge of $\Delta$ distinct from $D$, or $Q_{2}$ is a collapsing quadrilateral whose one spike is an edge of $\Delta$ not equal to $D$ and whose edges do not cross D.

Proof. Suppose that $\Delta$ is represented as a generalized quadrilateral by assigning to it the following vertices in the positive direction: $(a, b, b, v)$. Denoting the vertices of $Q_{2}$ by $x_{1} \leq x_{2} \leq x_{3} \leq x_{4}$ we may assume that

$$
a \leq x_{1} \leq b \leq x_{2} \leq b \leq x_{3} \leq v \leq x_{4} \leq a
$$

which implies $x_{2}=b$. Since $\overline{x_{2} x_{4}}$ is a critical chord then either $a=x_{4}$ or $v=x_{4}$. Clearly, the argument can be repeated in other cases too; it shows that $Q_{2}$ has a spike which is an edge of $\Delta$. In the degenerate case $Q_{2}$ coincides with an edge of $\Delta$; recall here, that by definition $Q_{2} \neq D$. If $Q_{2}$ is a triangle, then $Q_{2}=\Delta$, contradiction with the assumption that $Q_{1} \neq Q_{2}$. Now, suppose that $Q_{2}$ is a true quadrilateral. Then it cannot have $D$ as a spike. Hence its spike which is an edge of $\Delta$ must be either $\overline{a v}$ or $\overline{b v}$. Clearly, the edges of $Q_{2}$ cannot cross $D$.

It will be convenient to separate the following easy fact into a lemma.

Lemma 5.2. The sets $Q_{1}$ and $Q_{2}$ share at most one periodic vertex.

Proof. Indeed, otherwise the two shared vertices are not endpoints of $D$ and are not the endpoints of a critical chord. Hence if they are shared by $Q_{1}$ and $Q_{2}$ then $Q_{1}=Q_{2}$ contradicting the assumptions.

5.1. Slices of $\mathbb{L P}_{3}^{n p}$. For a family of chords $\mathcal{F}$, the equivalence relation $\sim_{\mathcal{F}}$ is defined as an equivalence relation on $\mathbb{S}$ under which two points of $\mathbb{S}$ are declared to be equivalent if and only if a finite chain of leaves of $\mathcal{F}$ connects them; we call the equivalence relation $\sim_{\mathcal{F}}$ generated by $\mathcal{F}$. We claim that each admissible qc-portrait QCP $\in \mathcal{S}_{D}$ is associated with a unique lamination denoted by $\sim_{\mathrm{QCP}}$. Indeed, by Theorem $\mathrm{A}$ there is a proper geolamination $\mathcal{L}_{\mathrm{QCP}}$ for which $\mathrm{QCP}$ is privileged; by definition, $\mathcal{L}_{\mathrm{QCP}}$ gives rise to an equivalence relation $\sim_{\mathcal{L}_{\mathrm{QCP}}}=\sim_{\mathrm{QCP}}$. By Theorem $\mathrm{B}$, $\sim_{\mathrm{QCP}}$ is unique for a given admissible qc-portrait QCP from $\mathcal{S}_{D}$. Indeed, if there are two distinct geolaminations $\mathcal{L}_{\mathrm{QCP}}$ and QCP is privileged for both of them, these two equal qc-portraits can be viewed as linked which implies that the laminations generated by the two proper geolaminations in question are the same (so that our notation $\sim_{\mathrm{QCP}}$ is appropriate). Set $\widehat{\mathcal{L}}_{\mathrm{QCP}}=\mathcal{L}_{\sim_{\mathrm{QCP}}}$. Denote by $\mathbb{L} \mathbb{P}_{3, D}^{n p}$ the family of all proper geolaminations with a critical leaf $D$ except for geolaminations of Siegel capture type. 
Since QCP $\in \mathcal{S}_{D}$, the endpoints $a$ and $b$ of $D$ are equivalent under $\sim_{\mathrm{QCP}}$. Now, if $\sim$ is a cubic lamination not of Siegel capture type, and $a$ and $b$ are $\sim$-equivalent, we can find a qc-portrait $\mathrm{QCP}=(Q, D)$ privileged for $\mathcal{L}_{\sim}$. Thus, the family $\mathrm{LAM}_{D}$ of laminations $\sim_{\mathrm{QCP}}$ obtained as described above for admissible qc-portraits QCP $\in \mathcal{S}_{D}$ is in fact the family of all cubic laminations $\sim$ not of Siegel capture type with $a \sim b$. Observe that if $\sim$ has a periodic Fatou gap $U$ of degree two then the non-degenerate refixed edge of $U$ is unique (if it exists) because the remap is of degree two and canonical geolaminations associated with laminations cannot have concatenations of edges on their boundaries. Also, if $\sim$ has a unique critical set then it has to coincide with the $\sim$-class of $a, b$ and is therefore finite.

Recall that every $\sigma_{2}$-invariant lamination $\sim$ has a unique minor set $m_{\sim}$ which is the convex hull of the image of a $\sim$-class of maximal diameter. By Thurston [Thu85], the minor sets of quadratic invariant laminations are pairwise disjoint and form a lamination of the unit circle. That is, minor sets of quadratic laminations form classes of QML. Domains of $\overline{\mathbb{D}} / \mathrm{QML}$ (i.e., components of $\overline{\mathbb{D}} / \mathrm{QML} \backslash \mathbb{S} / \mathrm{QML}$ ) come from critical sets of quadratic laminations $\sim$ which are periodic Fatou gaps $U$ of degree two; each such domain will be called the Main Cardioid of $U$. In particular, $U$ can coincide with the entire closed unit disk in which case we have the Main Cardioid of $\overline{\mathbb{D}}$ (or the Main Cardioid of the Mandelbrot set as it is usually called). In general $U$ can be of period $n$ in which case its Main Cardioid can be described as follows. Take all rotational sets $G$ of period $n$ inside $U$. Each such set has an unique longest edge denoted by $M_{G}$. This includes Siegel disks $S$ of period $n$ inside $U$ in which cases the critical edge of $S$ is its longest edge. Then the boundary of the Main Cardioid of $U$ is formed by sets $\sigma_{2}(M)$ taken over all rotational sets $G \subset U$ of period $n$.

Let us go back to the cubic case. Recall that $\mathrm{LAM}_{D}$ is the set of all $\sigma_{3^{-}}$ invariant laminations $\sim$ such that $a \sim b$ except for laminations of Siegel capture type, and that $\mathcal{S}_{D}$ is the family of all admissible qc-portraits $(Q, D)$ with $D$ as the second element. Each $(Q, D) \in \mathcal{S}_{D}$ is tagged by the chord or point (the minor) $\sigma_{3}(Q)$, and $\mathrm{CML}_{D}$ is the family of all such chords $\sigma_{3}(Q)$.

By Theorem A, every admissible qc-portrait is a privileged qc-portrait of a proper geolamination. Typically, but not always, an admissible qcportrait is privileged for a geolamination canonically associated with a cubic lamination from $\mathrm{LAM}_{D}$ (to each class of a lamination one associates its convex hull and then considers a geolamination formed by the edges of these convex hulls). Indeed, suppose that $\sim$ is a lamination from $\mathrm{LAM}_{D}$ which has a periodic critical Fatou gap $U$ of degree two and period $k$ with a refixed edge $M$ which is also an edge of an identity return triangle $T$, i.e. of a periodic triangle $T$ of period $k$. Denote the other two edges of $T$ by 
$\bar{m}$ and $\bar{n}$. If we remove the grand orbit of $M$ from $\widehat{\mathcal{L}}_{\sim}$, we will get a new proper geolamination $\mathcal{L}$ for which the gap $U$ becomes a new gap $V$ with refixed edges $\bar{m}$ and $\bar{n}$. The convex hulls of $\bar{m}(\bar{n})$ with their siblings are two admissible qc-portraits privileged for $\mathcal{L}$.

This shows that all edges of the triangle $\sigma_{3}(T)$ belong to $\mathrm{CML}_{D}$. However $\sigma_{3}(\bar{m})$ or $\sigma_{3}(\bar{n})$ are not minors of any geolaminations canonically associated to a lamination from $\mathrm{LAM}_{D}$. Call the triangle $T$ the major set of $\sim$; if such $T$ does not exist we call $M$ the major set of $U$. In other words, the major set of $\sim$ is the $\sim$-class of the refixed edge of $U$.

Definition 5.3 (Minor sets in the cubic case). For $\sim \in \mathrm{LAM}_{D}$ let $\mathfrak{g}_{D}$ be the $\sim$-class of $a$; moreover, if $\sim$ has a periodic Fatou gap $U$ of degree two, let $M_{\sim}$ be the major set of $U$. Let $C_{\sim}$ be either the first critical set of $\mathcal{L}_{\sim}$ (if it is different from $\mathfrak{g}_{D}$ and finite), the major set of $U$ (if the first critical set of $\sim$ is a periodic Fatou gap $U$ of degree two), or $\mathrm{CH}\left(\mathfrak{g}_{D}\right)$ (if $\sim$ has a unique critical class $\left.\mathfrak{g}_{D}\right)$. Set $\sigma_{3}\left(C_{\sim}\right)=m_{\sim}$ and call $m_{\sim}$ the minor set of $\sim$.

We are ready to prove Theorems $\mathrm{C}$ and $\mathrm{D}$.

Theorem 5.4. The family of chords $\mathrm{CML}_{D}$ is proper and gives rise to the lamination $\sim_{D}$. Convex hulls of $\sim_{D}$-classes are minor sets $m_{\sim}$ where $\sim$ belongs to $\mathrm{LAM}_{D}$.

Proof. Since by Theorem A the set $\mathcal{S}_{D}$ is compact, $\mathrm{CML}_{D}$ is a closed family of chords (minors). Consider the equivalence relation $\sim_{D}=\sim_{\mathrm{CML}_{D}}$. Clearly, $\sim_{D}$ is closed (because $\mathrm{CML}_{D}$ is closed). Assume that a lamination $\approx$ from $\mathrm{LAM}_{D}$ is a lamination such that its first critical set $C_{\approx}$ is a finite gap disjoint from $D$. Then we can insert collapsing quadrilaterals in $C_{\approx}$ in several ways and associate to it the corresponding proper geolaminations. Images of these collapsing quadrilaterals are minors of the corresponding proper geolaminations. Since these images are edges (and possibly some diagonals) of $m_{\approx}$, in this case $m_{\approx}$ is a class of equivalence of $\sim_{D}$.

Similar arguments show that the same holds if $\approx$ has a unique critical set coinciding with the convex hull $\mathrm{CH}\left(\mathfrak{g}_{\approx}\right)$ of the $\approx$-class $\mathfrak{g}_{\approx}$ of $a$. On the other hand, the analysis before Definition 5.3 shows that if the first critical set $C_{\approx}$ is a periodic Fatou gap of degree two then the corresponding class of equivalence of $\sim_{D}$ is the image $m_{\approx}$ of the major set $M_{\approx}$ of $\approx$. This covers all possibilities for the lamination $\approx$. Since each minor from $\mathrm{CML}_{D}$ can be associated with a proper geolamination and then with a lamination from $\mathrm{LAM}_{D}$, these cases cover the entire $\mathrm{CML}_{D}$ and complete the proof.

\section{REFERENCES}

[BFMOT13] A. Blokh, R. Fokkink, J. Mayer, L. Oversteegen, E. Tymchatyn, Fixed point theorems for plane continua with applications, Memoirs of the AMS 224 (2013). 
[BL02] A. Blokh, G. Levin, Growing trees, laminations and the dynamics on the Julia set, Ergodic Theory and Dynamical Systems 22 (2002), 63-97.

[BMOV13] A. Blokh, D. Mimbs, L. Oversteegen, K. Valkenburg, Laminations in the language of leaves, Trans. Amer. Math. Soc., 365 (2013), 5367-5391.

[BO08] A. Blokh, L. Oversteegen, Wandering Gaps for Weakly Hyperbolic Polynomials, in: "Complex dynamics: families and friends", ed. by D. Schleicher, A K Peters (2008), 139-168.

[BOPT14] A. Blokh, L. Oversteegen, R. Ptacek, V. Timorin, Combinatorial Models for Spaces of Cubic Polynomials, preprint arXiv:1405.4287 (2014)

[BOPT13a] A. Blokh, L. Oversteegen, R. Ptacek, V. Timorin, Laminations from the Main Cubioid, preprint arXiv:1305.5788 (2013)

[Chi07] D. Childers, Wandering polygons and recurrent critical leaves, Ergod. Th. and Dynam. Sys. 27 (2007), 87-107.

[CHMMO14] D. Cosper, J. Houghton, J. Mayer, L. Mernik, and J. Olson, Central Strips of Sibling Leaves in Laminations of the Unit Disk, preprint arXiv:1408.0223 (2014)

[Dou93] A. Douady, Descriptions of compact sets in $\mathbb{C}$, Topological methods in modern mathematics (Stony Brook, NY, 1991), ed. by L. Goldberg and A. Phillips, Publish or Perish, Houston, TX (1993), 429-465.

[Kiw02] J. Kiwi, Wandering orbit portraits, Trans. Amer. Math. Soc. 354 (2002), 14731485.

[Mil00] J. Milnor, Dynamics in One Complex Variable, Annals of Mathematical Studies 160, Princeton (2006).

[Sch04] D. Schleicher, On fibers and local connectivity of Mandelbrot and Multibrot sets, Fractal geometry and applications: a jubilee of Benoit Mandelbrot, ed. by M. Lapidus and M. van Frankenhuysen, Proc. Sympos. Pure Math. 72, Part 1, Amer. Math. Soc., Providence, RI (2004), 477-517.

[Sch09] D. Schleicher, Appendix: Laminations, Julia sets, and the Mandelbrot set, in: "Complex dynamics: Families and Friends", ed. by D. Schleicher, A K Peters (2009), 111-130.

[Thu85] W. Thurston. The combinatorics of iterated rational maps (1985), published in: "Complex dynamics: Families and Friends", ed. by D. Schleicher, A K Peters (2009), 1-108.

(Alexander Blokh and Lex Oversteegen) DEPARTMEnT OF MATHEMATICS, UniverSity of AlabAMA AT Birmingham, Birmingham, AL 35294

(Ross Ptacek and Vladlen Timorin) Faculty of Mathematics, LABORATORY of Algebraic Geometry and its Applications, Higher School of Economics, VAVILOVA ST. 7, 112312 Moscow, RuSsia

(Vladlen Timorin) IndePEndent University of Moscow, Bolshoy VlasyeVSKIY PEREUloK 11, 119002 Moscow, Russia

E-mail address, Alexander Blokh: ablokh@math . uab . edu

E-mail address, Lex Oversteegen: overstee@math. uab. edu

E-mail address, Ross Ptacek: rptacek@uab.edu

E-mail address, Vladlen Timorin: vtimorinehse.ru 